\title{
WELL-POSEDNESS AND SELF-SIMILAR ASYMPTOTICS FOR A THIN-FILM EQUATION*
}

\author{
MANUEL V. GNANN ${ }^{\dagger}$
}

\begin{abstract}
We investigate compactly supported solutions for a thin-film equation with linear mobility in the regime of perfect wetting. This problem has already been addressed by Carrillo and Toscani, proving that the source-type self-similar profile is a global attractor of entropy solutions with compactly supported initial data. Here we study small perturbations of source-type self-similar solutions for the corresponding classical free boundary problem and set up a global existence and uniqueness theory within weighted $L^{2}$-spaces under minimal assumptions. Furthermore, we derive asymptotics for the evolution of the solution, the free boundary, and the center of mass. As spatial translations are scaled out in our reference frame, the rate of convergence is higher than the one obtained by Carrillo and Toscani.
\end{abstract}

Key words. self-similar solutions, degenerate parabolic equations, fourth-order equations, nonlinear parabolic equations, free boundary problems, stability, asymptotic behavior of solutions, smoothness and regularity of solutions, uniqueness, classical solutions, thin fluid films, lubrication theory, Hele-Shaw flows, capillarity

AMS subject classifications. 35A02, 35A09, 35B35, 35B40, 35B65, 35C06, 35K25, 35K55, 35K65, 35R35, 76A20, 76D08, 76D27, 76D45

DOI. $10.1137 / 14099190 \mathrm{X}$

1. Introduction. We consider the following free boundary problem for the thinfilm equation:

$$
\begin{aligned}
\partial_{t} h+\partial_{z}\left(h \partial_{z}^{3} h\right) & =0 \quad \text { for } t>0 \text { and } z \in\left(Z_{-}(t), Z_{+}(t)\right), \\
h=\partial_{z} h & =0 \quad \text { for } t>0 \text { and } z=Z_{ \pm}(t), \\
\lim _{\left(Z_{-}(t), Z_{+}(t)\right) \ni z \rightarrow Z_{ \pm}(t)} \partial_{z}^{3} h & =\dot{Z}_{ \pm}(t) \quad \text { for } t>0, \\
h_{\mid t=0} & =h_{0} \quad \text { for } z \in\left(Z_{-}(0), Z_{+}(0)\right) .
\end{aligned}
$$

Equation (1.1a) is a fourth-order degenerate-parabolic equation modeling the film height $h=h(t, z) \geq 0$ of a $1+1$-dimensional liquid droplet as a function of time $t$ and base point $z[8,13,29] . Z_{ \pm}(t)$ denote the free boundaries of the problem. These represent the triple junctions between the three phases liquid, gas, and solid and correspond to the contact line in $2+1$ dimensions.

Since we are dealing with a fourth-order equation, two boundary conditions (1.1b) need to be imposed. $h=0$ at $z=Z_{ \pm}(t)$ just defines the contact line, while $\partial_{z} h=0$ at $z=Z_{ \pm}(t)$ implies that the slope of the film vanishes at the triple junction (zero contact angle). Assuming quasi-static motion, the contact angle is determined by a balance of surface tensions of the three interfaces (Young's law). In the case of $\partial_{z} h=0$ at $z=Z_{ \pm}(t)$ a global equilibrium is never achieved, that is, we are in the regime of perfect wetting.

* Received by the editors October 17, 2014; accepted for publication (in revised form) May 14, 2015; published electronically July 30, 2015. Funding of this work was provided by the International Max Planck Research School (IMPRS) of the Max Planck Institute for Mathematics in the Sciences (MPI MIS) in Leipzig and the Fields Institute in Toronto. The author's research was partially supported by the National Science Foundation under grant NSF DMS-1054115.

http://www.siam.org/journals/sima/47-4/99190.html

$\dagger$ Mathematics Department, University of Michigan, Ann Arbor, MI 48109-1043 (mvgnann@umich. edu). 
Since the boundary is a function of time $t$, we need a condition determining its evolution which is (1.1c). Viewing (1.1a) as a nonlinear continuity equation, we read off the horizontal fluid velocity as $v=\partial_{z}^{3} h$. By compatibility, this velocity has to match the speed of the contact lines at the free boundaries. A simple calculation shows that (1.1c) implies conservation of mass (volume) $M:=\int_{Z_{-}(t)}^{Z_{+}(t)} h(t, z) \mathrm{d} z$.

We mention that problem (1.1) can be derived by a lubrication approximation from Darcy's flow in the Hele-Shaw cell [18, 21, 22]. Equation (1.1a) is a specific case of a larger class of thin-film equations,

$$
\partial_{t} h+\partial_{z}\left(h^{n} \partial_{z}^{3} h\right)=0
$$

with a nonlinear mobility $h^{n}$ and where $n \in(0,3)$. Again, (1.2) can be derived from the Navier-Stokes system with an in general nonlinear slip condition at the liquid-solid interface by an asymptotic expansion [8, 13, 29].

Source-type self-similar solutions. It is instructive to study the case where the initial condition comes from a Dirac mass $M \delta_{0}$ at time $t=-1$, where $M$ is the mass of the droplet. Additionally we assume that the solution to (1.1) is self-similar. We note that (1.1a) remains invariant under the two-parameter scaling transformation

$$
(h, z, t) \mapsto\left(H_{*} h, Z_{*} z, H_{*}^{-1} Z_{*}^{4} t\right), \quad H_{*}, Z_{*}>0,
$$

that becomes a one-parameter family

$$
(h, z, t) \mapsto\left(Z_{*}^{-1} h, Z_{*} z, Z_{*}^{5} t\right), \quad Z_{*}>0,
$$

if we additionally assume conservation of mass, i.e., $H_{*} Z_{*}=1$. Hence our source-type self-similar solutions attain the structure

$$
h_{\mathrm{s}}(t, z)=(t+1)^{-\frac{1}{5}} H_{\mathrm{s}}(\xi), \quad \text { where } \xi=(t+1)^{-\frac{1}{5}} z .
$$

This ansatz automatically yields films for which the mass is constant in time $t$. We insert ansatz (1.3) in (1.1a) and obtain after some basic manipulations (cf. Appendix A)

$$
h_{s}(t, z)=\frac{15 M}{16} \frac{\left(1-x^{2}\right)^{2}}{(225 M(t+1) / 2)^{\frac{1}{5}}} \quad \text { for } x \in(-1,1) \text { with } \quad x=\frac{z}{(225 M(t+1) / 2)^{\frac{1}{5}}},
$$

which is known as the Smyth-Hill profile [34].

Convergence to source-type self-similar solutions. It is known that the long-time dynamics of (1.1) for rather general initial data is governed by the sourcetype self-similar solution (1.4). A first observation supporting this insight was obtained by Bernis $[3,4]$ through the study of the Cauchy problem for the thin-film equation in the $1+1$-dimensional case. Bernis was able to show that the speed of propagation of the interface is finite and the spreading rate asymptotically matches the one of the source-type solution. For the case of mobility exponent $n=1$ (the Darcy flow in the Hele-Shaw cell), a stronger result by Carrillo and Toscani is available [12]: Using analogies to the second-order counterpart of (1.1a), the porous medium equation [11], the authors show that entropy solutions with compactly supported initial data or initial data with finite second moment converge to the source-type solution 
with the same mass in $L^{1}(\mathbb{R})$, where the rate is given by $(t+1)^{-\frac{1}{5}}$. This rate also applies if the solution is written in self-similar variables

$$
H(t, \xi):=(t+1)^{\frac{1}{5}} h\left(t,(t+1)^{\frac{1}{5}} \xi\right) \quad \text { for } \xi \in \mathbb{R},
$$

in which - in view of (1.3) - the source-type solution is stationary. The result was upgraded to convergence in $H^{1}(\mathbb{R})$ with a suboptimal rate by Carlen and Ulusoy [10]. Subsequently, Matthes, McCann, and Savaré [25] improved this result to the $d$-dimensional thin-film equation and convergence in $H^{1}\left(\mathbb{R}^{d}\right)$ with the optimal rate $(t+1)^{-\frac{1}{5}}$ in self-similar variables (1.5) (cf. also Carlen [9]). By the standard embedding $H^{1}(\mathbb{R}) \hookrightarrow L^{p}(\mathbb{R})$ with $1 \leq p \leq \infty$, the latter result also implies convergence in $L^{p}(\mathbb{R})$ with rate $(t+1)^{-\frac{1}{5}}$. We also refer to the work of Bernoff and Witelski [6], explicitly solving the linear evolution for $n=1$ in the $1+1$-dimensional case and providing a numerical analysis for other $n$. The work of McCann and Seis [26] generalizes the linear analysis to higher dimensions and more general fourth-order equations by exploiting the gradient flow structure of the thin-film equation and relying on the analogous analysis for the porous medium equation [33].

Cauchy problem and linearization. We consider perturbations of source-type solutions (1.4), for which we introduce volumetric (mass-Lagrangian) coordinates as follows:

$$
\int_{Z_{-}(t)}^{Z(t, x)} h(t, z) \mathrm{d} z:=\frac{15 M}{16} \int_{-1}^{x}\left(1-\left(x^{\prime}\right)^{2}\right)^{2} \mathrm{~d} x^{\prime} \quad \text { for } x \in(-1,1) .
$$

This equality, together with (1.1), implicitly defines $Z(t, x)$. One advantage of using (1.6) is that in the new coordinates the boundary is fixed to the points $x= \pm 1$. Additionally, the source-type self-similar solution in these coordinates is given by (cf. (1.4))

$$
Z_{\mathrm{s}}(t, x)=\left(\frac{225 M(t+1)}{2}\right)^{\frac{1}{5}} x
$$

i.e., it is a linear function in $x$. Furthermore, the transformation guarantees that the mass $M$ is conserved in time $t$. This condition would not necessarily be fulfilled if we chose a related coordinate transformation, the hodograph transform (cf. [14]) where $h(t, Z(t, x)):=h_{\mathrm{s}}\left(t, Z_{\mathrm{s}}(t, x)\right)$. Also note that the latter transformation would fix the maximal height of any perturbation to match the one of $h_{0}(t, z)$, clearly being an unphysical assumption.

In order to derive an evolution equation in the new coordinates, we first differentiate (1.6) with respect to $x$ and obtain the relation

$$
h(t, Z(t, x)) \partial_{x} Z(t, x)=\frac{15 M}{16}\left(1-x^{2}\right)^{2} .
$$

Furthermore, differentiating (1.6) with respect to $t$ leads to

$$
h(t, Z(t, x)) \partial_{t} Z(t, x)+\int_{Z_{-}(t)}^{Z(t, x)} \partial_{t} h(t, z) \mathrm{d} z=0,
$$

which by using (1.1a) yields

$$
h(t, Z(t, x)) \partial_{t} Z(t, x)-\int_{Z_{-}(t)}^{Z(t, x)} \partial_{z}\left(h \partial_{z}^{3} h\right)(t, z) \mathrm{d} z=0 .
$$


We integrate the second integral and observe that due to the first boundary condition in (1.1b) and condition (1.1c), the boundary term at $z=Z_{-}(t)$ vanishes. Thus we arrive at the relation

$$
\partial_{t} Z(t, x)=\partial_{z}^{3} h(t, Z(t, x)) .
$$

Due to (1.8) it is convenient to formulate the equation in terms of

$$
G:=\frac{1}{\partial_{x} Z} .
$$

Then the derivatives are transformed as $\partial_{z}=G \partial_{x}$ which, together with (1.8) and (1.9), demonstrates that

$$
\partial_{t} Z(t, x)=\left(G(t, x) \partial_{x}\right)^{3} G(t, x) \frac{15 M}{16}\left(1-x^{2}\right)^{2} .
$$

Since by the chain rule $\partial_{t} G(t, x)=-(G(t, x))^{2} \partial_{x} \partial_{t} Z(t, x)$, we obtain the evolution equation for $G$ given by

$$
\partial_{t} G=-\frac{15 M}{16} G^{2} \partial_{x}\left(G \partial_{x}\right)^{3}\left(1-x^{2}\right)^{2} G \quad \text { for } t>0 \text { and }-1<x<1 .
$$

The source-type solution for $G$ reads

$$
G_{\mathrm{S}}(t, x):=\frac{1}{\partial_{x} Z_{\mathrm{s}}(t, x)} \stackrel{(1.7)}{=}\left(\frac{225 M(t+1)}{2}\right)^{-\frac{1}{5}}
$$

For convenience we apply a further transformation and factor off $G_{\mathrm{s}}$

$$
G(t, x)=: G_{\mathrm{S}}(t, x) F(t, x)=\left(\frac{225 M(t+1)}{2}\right)^{-\frac{1}{5}} F(t, x) .
$$

A simple calculation shows that the evolution equation (1.12) then transforms into

$$
120(t+1) \partial_{t} F=24 F-F^{2} \partial_{x}\left(F \partial_{x}\right)^{3}\left(1-x^{2}\right)^{2} F \quad \text { for } t>0 \text { and }-1<x<1 .
$$

It is handy to introduce the logarithmic time variable

$$
\tau:=\frac{\ln (t+1)}{120},
$$

implying $120(t+1) \partial_{t}=\partial_{\tau}$, and to consider $F$ as a function of $\tau$ and $x$. Then (1.14) transforms into the not explicitly time-dependent equation

$$
\partial_{\tau} F=24 F-F^{2} \partial_{x}\left(F \partial_{x}\right)^{3}\left(1-x^{2}\right)^{2} F \quad \text { for } \tau>0 \text { and }-1<x<1 .
$$

Thus in terms of $F$ the source-type self-similar solution is merely the constant function $F_{\mathrm{s}}=1$. Since we want to study perturbations of $F_{\mathrm{s}}=1$, we set

$$
u:=F-1 .
$$

We observe that, using (1.17), the right-hand side of (1.16) is a linear combination (with constant coefficients) of terms of the form

$$
\partial_{x}^{\ell_{0}}\left(1-x^{2}\right)^{2} \times \prod_{j=1}^{m} \partial_{x}^{\ell_{j}} u \quad \text { with } m \in\{1, \ldots, 6\} \text { and } \sum_{j=0}^{m} \ell_{j}=4 \text {, }
$$


where we can assume $m \geq 1$ since the source-type solution $u_{\mathrm{s}}=0$ solves (1.16). A more detailed computation (contained in Appendix A) demonstrates that the linear part $-\mathcal{L}$ of the right-hand side in (1.16) is given by

$$
\begin{aligned}
\mathcal{L} & =\left(1-x^{2}\right)^{2} \partial_{x}^{4}-20 x\left(1-x^{2}\right) \partial_{x}^{3}+40\left(3 x^{2}-1\right) \partial_{x}^{2}+240 x \partial_{x}+120 \\
& =\frac{1}{\left(1-x^{2}\right)^{3}} \partial_{x}^{2}\left(1-x^{2}\right)^{5} \partial_{x}^{2}-\frac{30}{\left(1-x^{2}\right)^{3}} \partial_{x}\left(1-x^{2}\right)^{4} \partial_{x}+120
\end{aligned}
$$

We are thus lead to study the Cauchy problem

$$
\begin{aligned}
\partial_{\tau} u+\mathcal{L} u & =\mathcal{N}(u) \text { for } \tau>0 \text { and }-1<x<1, \\
u_{\mid \tau=0} & =u_{0} \text { for }-1<x<1 .
\end{aligned}
$$

Here $u_{0}$ denotes the initial data and the nonlinearity $\mathcal{N}(u)$ is a linear combination (with constant coefficients) of terms of the form (cf. (1.18))

$$
\partial_{x}^{\ell_{0}}\left(1-x^{2}\right)^{2} \times \prod_{j=1}^{m} \partial_{x}^{\ell_{j}} u \quad \text { with } m \in\{2, \ldots, 6\} \text { and } \sum_{j=0}^{m} \ell_{j}=4 .
$$

A precise definition (which is immaterial for the subsequent analysis) is given in (A.5).

We note that $\mathcal{L}$ can be rewritten as $\mathcal{L}=\mathcal{A}(\mathcal{A}+2)$ with the degenerate-parabolic second-order operator

$$
\mathcal{A}:=-\frac{1}{\left(1-x^{2}\right)^{3}} \partial_{x}\left(1-x^{2}\right)^{4} \partial_{x}+10
$$

A similar structure has been found by Bernoff and Witelski in [6, section 5], however, with a different second-order operator (which is due to a differently chosen coordinate system). The operator $\mathcal{A}-3$ is known as a special version of the Gegenbauer differential operator [35]. Interestingly, $\mathcal{L}$ cannot be written as the square of a second-order operator as in a work by Giacomelli, Knüpfer, and Otto [17], treating perturbations of a parabola profile in the case of the half space. We emphasize that - despite (1.20) is structurally more complicated - the subsequent analysis has some similarities to [17], which is why we keep the presentation brief in those cases, where the analogies are apparent.

We would like to point out that it may seem surprising that no constraint at the boundary of the domain has to be fulfilled, although we deal with a fourth-order problem. Yet, notice that the linear operator $\mathcal{L}$ is degenerate due to the prefactors $\left(1-x^{2}\right)$, which is why no boundary condition has to be imposed in order to obtain a well-posed problem. Our sequence of transformations (cf. (1.6), (1.10), (1.13), (1.15), and (1.17)) is chosen such that the boundary conditions (1.1b) and (1.1c) of the original problem (1.1) are automatically fulfilled.

In view of (1.20), it is natural to study the linear Cauchy problem

$$
\begin{aligned}
\partial_{\tau} u+\mathcal{L} u & =f \quad \text { for } \tau \in I \text { and }-1<x<1, \\
u_{\mid \tau=0} & =u_{0} \quad \text { for }-1<x<1,
\end{aligned}
$$

where $I=\left(0, \tau_{1}\right) \subset(0, \infty)$ is an interval. The above discussions on the boundary conditions apply to (1.23) as well. 
Notation. Throughout the paper we will write $f \lesssim_{S} g$ whenever a constant $C \geq 1$, depending on the set of parameters $S$, exists such that $f \leq C g$. We write $f \sim_{S} g$ if $f \lesssim_{S} g$ and $g \lesssim_{S} f$. We say that a property is true for $x \gg_{S} 1\left(x \ll_{S} 1\right)$, whenever a constant $C>0$, depending on parameters $S$, exists such that the property is true for all $x$ with $x \geq C(x \leq C)$. If $S=\emptyset$ we just write $f \lesssim g$, etc.

Weighted Sobolev spaces and their interpolation spaces. For establishing existence and uniqueness of (1.20), respectively, (1.23), we introduce a scale of weighted Sobolev spaces. In Corollary 4.3 we prove that the operator $\mathcal{L}$ is symmetric with respect to the bilinear forms

$$
\left\langle u_{1}, u_{2}\right\rangle_{k}:=\int_{-1}^{1}\left(1-x^{2}\right)^{3+k}\left(\partial_{x}^{k} u_{1}\right)\left(\partial_{x}^{k} u_{2}\right) \mathrm{d} x, \quad \text { where } k \geq 0,
$$

and $u_{1}, u_{2} \in C^{\infty}([-1,1])$. The induced seminorms are denoted by $[\cdot]_{k}$ and it is convenient to introduce the summed seminorms $[\cdot]_{\ell, k}$ (with $k \geq \ell \geq 0$ ) through

$$
[u]_{\ell, k}^{2}:=\sum_{m=\ell}^{k}[u]_{m}^{2}
$$

The bilinear forms induce a scalar product through

$$
\left(u_{1}, u_{2}\right)_{k}:=\sum_{j=0}^{k}\left\langle u_{1}, u_{2}\right\rangle_{j} \stackrel{(1.24)}{=} \sum_{j=0}^{k} \int_{-1}^{1}\left(1-x^{2}\right)^{j+3}\left(\partial_{x}^{j} u_{1}\right)\left(\partial_{x}^{j} u_{2}\right) \mathrm{d} x .
$$

We denote the induced norm by $|\cdot|_{k}$ and we also use the spaces

$\mathcal{H}^{k}$ defined as the completion of $\left\{u \in C^{\infty}([-1,1]):|u|_{k}<\infty\right\}$ with respect to $|\cdot|_{k}$.

In order to prove well-posedness of (1.20), respectively, (1.23), estimating the nonlinearity $\mathcal{N}(u)$ requires at least control of $\|u\|_{C^{0}([-1,1])}$. Due to the scaling of the seminorms $[\cdot]_{k}$ close to the boundaries $x= \pm 1$, this demands at least control of the initial data $u_{0}$ in the norm $|\cdot|_{4}$. However, the estimate $\|u\|_{C^{0}([-1,1])} \lesssim|u|_{4}$ fails due to logarithmic corrections. ${ }^{1}$ This requires us to introduce interpolation spaces of $\mathcal{H}^{k}$ (compare to [17, sections 1 and 6]).

Definition 1.1. For $k \geq 1$ we define the interpolation norms

$$
|u|_{k, *}:=\int_{0}^{\infty} \inf _{\substack{u=u_{-}+u_{+} \\ u_{ \pm} \in \mathcal{H}^{k \pm 1}}}\left(\sigma^{-1}\left|u_{-}\right|_{k-1}^{2}+\sigma\left|u_{+}\right|_{k+1}^{2}\right)^{\frac{1}{2}} \frac{\mathrm{d} \sigma}{\sigma} .
$$

The corresponding function spaces $\mathcal{H}_{*}^{k}$ are defined as the completion of

$$
\left\{u \in C^{\infty}([-1,1]):|u|_{k, *}<\infty\right\} \text { with respect to }|\cdot|_{k, *} .
$$

Our definition of the interpolation space relies on the $K$-method of interpolation theory, that is, we have $\mathcal{H}_{*}^{k}=\left(\mathcal{H}^{k-1}, \mathcal{H}^{k+1}\right)_{\frac{1}{2}, 1}$, where $(\cdot, \cdot)_{\alpha, q}$ denotes the real interpolation functor (cf. [1, Chapter 7]). In fact, for the interpolated spaces our desired

\footnotetext{
${ }^{1} \mathrm{~A}$ counterexample is given by $u(x):=\int_{0}^{x} \int_{0}^{x_{1}} \int_{0}^{x_{2}} \int_{0}^{x_{3}} \frac{\eta\left(x_{4}\right) \mathrm{d} x_{4} \mathrm{~d} x_{3} \mathrm{~d} x_{2} \mathrm{~d} x_{1}}{\left(1-x_{4}^{2}\right)^{4}\left|\ln \left(1-x_{4}^{2}\right)\right|}$, where $\eta \in$ $C^{\infty}([-1,1])$ with $\eta(x) \equiv 0$ for $x \leq \frac{1}{4}$ and $\eta(x) \equiv 1$ for $x \geq \frac{3}{4}$.
} 
estimate $\|u\|_{C^{0}([-1,1])} \lesssim|u|_{4, *}$ holds true (cf. Lemma 3.5). It will turn out to be a consequence of the standard embedding $B_{1}^{\frac{1}{2}, 2}((-1,1)) \hookrightarrow C^{0}([-1,1])$ for Besov spaces, for which the fine index 1 is crucial.

For the treatment of the parabolic equation, we introduce the notation $L_{\mu}^{2}(I ; X)$ (where $X$ is a Hilbert space, $I \subseteq(0, \infty)$, and $\mu \in \mathbb{R}$ ) denoting the space of all measurable $u: I \rightarrow X$ such that $\left(\tau \mapsto e^{-\mu \tau} u(\tau)\right) \in L^{2}(I ; X)$. The spaces $C_{\mu}^{0}(\bar{I} ; X)$ and $H_{\mu}^{1}(I ; X)$ are defined analogously. In the same way as in Definition 1.1, we define the interpolation norms

$$
\|u\|_{L_{\mu}^{2}\left(I ; \mathcal{H}^{k}\right)^{*}}:=\int_{0}^{\infty} \inf _{u=u_{-}+u_{+}}\left(\sigma^{-1}\left\|u_{-}\right\|_{L_{\mu}^{2}\left(I ; \mathcal{H}^{k-1}\right)}^{2}+\sigma\left\|u_{+}\right\|_{L_{\mu}^{2}\left(I ; \mathcal{H}^{k+1}\right)}^{2}\right)^{\frac{1}{2}} \frac{\mathrm{d} \sigma}{\sigma},
$$

$$
\|u\|_{H_{\mu}^{1}\left(I ; \mathcal{H}^{k}\right)^{*}}:=\int_{0}^{\infty} \inf _{u=u_{-}+u_{+}}\left(\sigma^{-1}\left\|u_{-}\right\|_{H_{\mu}^{1}\left(I ; \mathcal{H}^{k-1}\right)}^{2}+\sigma\left\|u_{+}\right\|_{H_{\mu}^{1}\left(I ; \mathcal{H}^{k+1}\right)}^{2}\right)^{\frac{1}{2}} \frac{\mathrm{d} \sigma}{\sigma},
$$

and

$$
\|u\|_{C_{\mu}^{0}\left(\bar{I} ; \mathcal{H}^{k}\right)^{*}}:=\int_{0}^{\infty} \inf _{u=u_{-}+u_{+}}\left(\sigma^{-1}\left\|u_{-}\right\|_{C_{\mu}^{0}\left(\bar{I} ; \mathcal{H}^{k-1}\right)}^{2}+\sigma\left\|u_{+}\right\|_{C_{\mu}^{0}\left(\bar{I} ; \mathcal{H}^{k+1}\right)}^{2}\right)^{\frac{1}{2}} \frac{\mathrm{d} \sigma}{\sigma}
$$

with corresponding spaces.

2. The main results. For our minimal existence and uniqueness result we introduce the notation

$$
\|u\|:=\|u\|_{H^{1}\left((0, \infty) ; \mathcal{H}^{2}\right)^{*} \cap C^{0}\left([0, \infty) ; \mathcal{H}^{4}\right)^{*} \cap L^{2}\left((0, \infty) ; \mathcal{H}^{6}\right)^{*}}
$$

and $\left\|u_{0}\right\|_{0}:=\left|u_{0}\right|_{4, *}$.

THEOREM 2.1. There exists a universal constant $\delta>0$ such that for every initial value $u_{0} \in \mathcal{H}_{*}^{4}$ with $\left\|u_{0}\right\|_{0} \leq \delta$, problem (1.20) has a unique solution

$$
u \in H^{1}\left((0, \infty) ; \mathcal{H}^{2}\right)^{*} \cap C^{0}\left([0, \infty) ; \mathcal{H}^{4}\right)^{*} \cap L^{2}\left((0, \infty) ; \mathcal{H}^{6}\right)^{*} .
$$

This solution obeys the a priori estimate $\|u\| \lesssim\left\|u_{0}\right\|_{0}$. Furthermore, for all $\mu<120$

$$
|u(\tau)|_{4, *} \lesssim \mu e^{-\mu \tau} \quad \text { for } \tau \geq 0 .
$$

In fact, we can even show the following.

ThEOREM 2.2. In the situation of Theorem 2.1, for any $k \geq 2$ there exists $\tau_{k} \geq 0$ such that for any $\mu<120$

$$
u_{\mid\left[\tau_{k}, \infty\right)} \in C_{\mu}^{0}\left(\left[\tau_{k}, \infty\right) ; \mathcal{H}_{*}^{k+2}\right) \quad \text { and } \quad|u(\tau)|_{k+2, *} \lesssim_{\mu, k} e^{-\mu \tau} \text { for } \tau \geq \tau_{k} .
$$

Comparison to other well-posedness results. To our knowledge, Theorem 2.1 is the first global existence and uniqueness result for a thin-film equation with compactly supported initial data. A corresponding short-time well-posedness result (for initial data that are not necessarily close to a source-type solution) was obtained by Giacomelli and Knüpfer using Schauder estimates in weighted Hölder spaces [16].

The result may also be considered as a first step toward a uniqueness proof for small perturbations of source-type solutions within the class of entropy (or strong) 
solutions - a question already raised in [12]. Here, uniqueness of solutions under the weaker assumption $\left\|u_{0}\right\|_{C^{0}([-1,1])} \ll 1$ is presumably true. We expect that a proof of such a result should be possible using recent ideas of John [20]. The latter rely on the theory of singular integral operators in weighted $L^{p}$-spaces which have been first applied by Koch to the porous medium equation [23]. However, the linear analysis, presented in section 4, will be more subtle in this case and probably requires detailed (pointwise) Gaussian estimates of the parabolic Green's function (or a localization argument in order to utilize John's result directly) rather than $L^{2}$-integral control of the solution only.

Remark 2.3. Notably, Theorem 2.2 does not yield that the solution $u(\tau, x)$ is instantaneously smooth for $\tau>0$. Such a statement can be proved in case of perturbations of a parabola [17, Theorems 1.2 and 1.3]. However, it appears that the proof strategy of [17] cannot be applied directly.

Comparison to other convergence results. With the help of Lemma 3.5 and going back to the original time variable (cf. (1.15)), estimates (2.1) and (2.2) imply the following: For every $\varepsilon>0$ and every $\ell \geq 0$, there exists $t_{\ell} \geq 0$ (where $t_{0}=0$ ) with

$$
\left|\partial_{x}^{\ell} u(t, x)\right| \lesssim_{\varepsilon, \ell}(t+1)^{-1+\varepsilon} \text { for all } x \in[-1,1] \text { and } t \geq t_{\ell} .
$$

Using estimate (2.3) in (1.20a), an induction argument (iteratively differentiating (1.20a) in time) also leads to the following statement: For any $\varepsilon>0$ and all $k, \ell \geq 0$, there exists $t_{k, \ell} \geq 0$ with

$$
(t+1)^{k}\left|\partial_{t}^{k} \partial_{x}^{\ell} u(t, x)\right| \lesssim_{\varepsilon, k, \ell}(t+1)^{-1+\varepsilon} \quad \text { for all } x \in[-1,1] \text { and } t \geq t_{k, \ell} .
$$

We notice that the decay with rate $(t+1)^{-1+0}$ is faster than with the rate $(t+1)^{-\frac{1}{5}}$ obtained by Carrillo and Toscani [12], respectively, Matthes, McCann, and Savaré [25]. Our new result is complementary to [12, 25]: As it was pointed out by Bernoff and Witelski [6], respectively, McCann and Seis [26] for the linearized problem, the rate $(t+1)^{-\frac{1}{5}}$ can be observed in the self-similar variables (1.5). This slower rate is due to translations in space. In our setting the accelerated convergence is due to the fact that spatial translations are not seen in the transformed set of variables: Clearly, a (time-dependent) shift $\tilde{h}(t, z):=h(t, z+\alpha(t))$ in the lateral variable of a given profile $h=h(t, z)$ leads to a shift $\tilde{Z}(t, x)=Z(t, x)+\alpha(t)$ in the volumetric coordinates defined through (1.6). In view of transformation (1.10), we have $\tilde{G}(t, x)=1 / \partial_{x} \tilde{Z}(t, x)=1 / \partial_{x} Z(t, x)=G(t, x)$, i.e., the $G$-coordinates and-applying the subsequent transformations (1.13) and (1.17) - also the $u$-coordinates are the same. Hence, translations in time (with rate $(t+1)^{-1}$; cf. [6]) are limiting. This is reflected in the spectral gap of the linear operator $\mathcal{L}$ (cf. section 4). The smallness assumption $\left\|u_{0}\right\|_{0} \ll 1$ leads to the fact that the linearization of (1.20) dominates the evolution and $u_{0}$ will be in the domain of attraction of a fixed point problem (cf. sections 5 and 6 ).

Transformation into the original variables. We utilize the representation (1.8) for the film height $h$ in conjunction with transformations (1.10), (1.13), and (1.17) to obtain

$$
h(t, Z(t, x))=\frac{15 M}{16}\left(1-x^{2}\right)^{2}\left(\frac{225 M(t+1)}{2}\right)^{-\frac{1}{5}}(1+u(t, x)) \quad \text { for } x \in[-1,1]
$$


and $t \geq 0$, or-using (1.4) and (1.7)-equivalently

$$
h(t, Z(t, x))-h_{\mathrm{s}}\left(t, Z_{\mathrm{s}}(t, x)\right)=\frac{15 M}{16}\left(1-x^{2}\right)^{2}\left(\frac{225 M(t+1)}{2}\right)^{-\frac{1}{5}} u(t, x)
$$

for $x \in[-1,1]$ and $t \geq 0$. In view of $(2.4)$, this already implies convergence with a faster rate, i.e.,

$(t+1)^{\frac{1}{5}+k}\left|\partial_{t}^{k} \partial_{x}^{\ell}\left(h(t, Z(t, x))-h_{\mathbf{s}}\left(t, Z_{\mathbf{s}}(t, x)\right)\right)\right| \lesssim_{\varepsilon, k, \ell}(t+1)^{-1+\varepsilon} \quad$ for all $x \in[-1,1]$

and $t \geq t_{k, \ell}$, where $t_{k, \ell} \geq 0$ is the same as in (2.4) (in particular $t_{0,0}=0$ ), $\varepsilon>0$, and $k, \ell \geq 0$. Not surprisingly, the decay rate is higher than for the known results $[12,25]$ in the self-similar variables $(1.5)$, as we compare the solutions $h(t, z)$ and $h_{\mathrm{S}}\left(t, z^{\prime}\right)$ at different (time-dependent) positions $z=Z(t, x)$, respectively, $z^{\prime}=Z_{\mathrm{s}}(t, x)$. Nevertheless, (2.6) is nontrivial as we can conclude, for instance, that

$$
\left|\max _{z \in \mathbb{R}} h(t, z)-\max _{z^{\prime} \in \mathbb{R}} h_{\mathrm{s}}\left(t, z^{\prime}\right)\right|
$$

decays with rate $(t+1)^{-1+0}$ and not $(t+1)^{-\frac{1}{5}}$.

In order to obtain control on the volumetric coordinates $Z(t, x)$ and in particular the speed $\dot{Z}_{ \pm}(t)=\partial_{t} Z(t, \pm 1)$, we employ transformations (1.13) and (1.17) in (1.11) and arrive at

$$
\partial_{t} Z(t, x)=\frac{15 M}{16}\left(\frac{225 M(t+1)}{2}\right)^{-\frac{4}{5}}\left((1+u(t, x)) \partial_{x}\right)^{3}\left(1-x^{2}\right)^{2}(1+u(t, x))
$$

for $x \in(-1,1)$ and $t \geq 0$. Setting $u:=u_{\mathrm{s}}=0,(2.7)$ reduces to the self-similar solution $\partial_{t} Z_{\mathrm{s}}(t, x)$ (cf. (1.7)), so that the difference $(t+1)^{\frac{4}{5}}\left(\partial_{t} Z-\partial_{t} Z_{\mathrm{s}}\right)$ is a linear combination (with constant coefficients) of terms of the form

$$
\partial_{x}^{\ell_{0}}\left(1-x^{2}\right)^{2} \times \prod_{j=1}^{m} \partial_{x}^{\ell_{j}} u \quad \text { with } m \in\{1, \ldots, 4\} \text { and } \sum_{j=0}^{m} \ell_{j}=3 .
$$

Utilizing (2.4) once more, we infer that there exist $t_{k, \ell}^{\prime} \geq 0$ such that

$$
(t+1)^{\frac{4}{5}+k}\left|\partial_{t}^{k+1} \partial_{x}^{\ell}\left(Z(t, x)-Z_{\mathbf{s}}(t, x)\right)\right| \lesssim_{k, \ell, \varepsilon}(t+1)^{-1+\varepsilon} \quad \text { for } x \in[-1,1]
$$

and $t \geq t_{k, \ell}^{\prime}$, where $\varepsilon>0$ and $k, \ell \geq 0$.

Remark 2.4. From (2.8) we obtain relatively explicit asymptotic expressions for the speed of the contact points (cf. (1.4) and (1.7))

$$
\dot{Z}_{ \pm}(t)= \pm \frac{1}{5}\left(\frac{225 M}{2}\right)^{\frac{1}{5}}(t+1)^{-\frac{4}{5}}\left(1+O\left((t+1)^{-1+0}\right)\right) \quad \text { as } t \rightarrow \infty .
$$

Equation (2.9) is more precise than the findings of Bernis in [3, Theorems 7.1 and 7.2 ], where only upper and lower bounds with rate $\sim(t+1)^{\frac{1}{5}}$ for the growth of the support have been derived.

Using the transformations in (1.10), (1.13), and (1.17) together with the representation of the source-type solution (1.7), we further obtain

$$
\partial_{x} Z-\partial_{x} Z_{\mathrm{s}}=-\left(\frac{225 M(t+1)}{2}\right)^{\frac{1}{5}} \frac{u}{1+u} .
$$


With the help of (2.4) and integrating once in $x$, we get

$$
(t+1)^{-\frac{1}{5}+k}\left|\partial_{t}^{k} \partial_{x}^{\ell}\left(Z(t, x)-Z_{\mathrm{s}}(t, x)-\alpha(t)\right)\right| \lesssim_{\varepsilon, k, \ell}(t+1)^{-1+\varepsilon} \text { for } x \in[-1,1]
$$

and $t \geq t_{k, \ell}$, where $k, \ell \geq 0, t_{k, \ell}$ is chosen as in (2.4), and

$$
\begin{aligned}
\alpha(t) & :=\frac{1}{M} \int_{Z_{-}(t)}^{Z_{+}(t)} z h(t, z) \mathrm{d} z=\frac{1}{M} \int_{-1}^{1} Z(t, x) h(t, Z(t, x)) \partial_{x} Z(t, x) \mathrm{d} x \\
& \stackrel{(1.8)}{=} \frac{15}{16} \int_{-1}^{1} Z(t, x)\left(1-x^{2}\right)^{2} \mathrm{~d} x
\end{aligned}
$$

is the center of mass. The decay of $\alpha(t)$ follows from (2.8), that is,

$$
(t+1)^{-\frac{1}{5}+k}\left|\frac{\mathrm{d}^{k}}{\mathrm{~d} t^{k}}(\alpha(t)-\alpha(\infty))\right| \lesssim_{k, \varepsilon}(t+1)^{-1+\varepsilon} \quad \text { for } t \geq t_{k}^{\prime}
$$

with $k \geq 0, \varepsilon>0$, and some $t_{k}^{\prime} \geq 0$.

Remark 2.5. The meaning of the assumption $\left|u_{0}\right|_{4, *} \ll 1$ (that is, in particular $\left\|u_{0}\right\|_{C^{0}([-1,1])} \ll 1$ by Lemma 3.5$)$ for the initial data in terms of the original variables may be best understood by appealing to (2.5), i.e.,

$$
h\left(t, Z_{0}(x)\right)-h_{\mathrm{s}}\left(0, Z_{\mathrm{s}}(0, x)\right)=\frac{15 M}{16}\left(1-x^{2}\right)^{2}\left(\frac{225 M}{2}\right)^{-\frac{1}{5}} u_{0}(x) \quad \text { for } x \in[-1,1],
$$

and noting that due to (2.10) the Lipschitz constant of $Z_{0}-Z_{\mathrm{s} \mid \mathrm{t}=0}$, where $Z_{0}(x):=$ $Z(0, x)$, is small.

We can use the transformation of derivatives $\partial_{x} \mapsto \partial_{x} Z \partial_{z}$ and $\partial_{t} \mapsto \partial_{t}+\partial_{t} Z \partial_{z}$, respectively, $\partial_{x} \mapsto \partial_{x} Z_{\mathrm{s}} \partial_{z}$ and $\partial_{t} \mapsto \partial_{t}+\partial_{t} Z_{\mathrm{s}} \partial_{z}$ in (2.6). With the help of the decay estimates for the volumetric coordinates in (2.8) and (2.11), and the explicit expressions for the source-type solution in (1.4) and (1.7), we obtain

$$
(t+1)^{k+\frac{\ell+1}{5}}\left|\partial_{t}^{k} \partial_{z}^{\ell} h(t, Z(t, x))-\partial_{t}^{k} \partial_{z}^{\ell} h_{\mathrm{s}}\left(t, Z_{\mathrm{s}}(t, x)\right)\right| \lesssim_{\varepsilon, k, \ell}(t+1)^{-1+\varepsilon}
$$

for $x \in[-1,1], t \geq t_{k, \ell}^{\prime \prime}$ (with some $t_{k, \ell}^{\prime \prime} \geq 0$ ), $\varepsilon>0$, and $k, \ell \geq 0$. Equation (2.15) is an improvement of the previous results $[12,25]$ in the sense that also convergence of higher derivatives is shown. This is due to the fact that we are considering a classical free boundary problem: Second and higher derivatives are defined in the support of the droplet, but have discontinuities if studied on the real line as in $[12,25]$.

As a last step, we compare the solutions $h=h(t, z)$ and $h_{\mathrm{s}}=h_{\mathrm{s}}(t, z)$ at the same position $z$. First observe that for $\ell \in\{0,1\}$

$$
\begin{aligned}
\left|\partial_{z}^{\ell} h_{\mathrm{s}}(t, Z(t, x))-\partial_{z}^{\ell} h_{\mathrm{s}}\left(t, Z_{\mathrm{s}}(t, x)\right)\right| \leq & \left\|\partial_{z}^{\ell+1} h_{\mathrm{s}}(t, \cdot)\right\|_{C^{0}\left(\left[Z_{-}(t), Z_{+}(t)\right]\right)} \\
& \times\left|Z(t, x)-Z_{\mathrm{s}}(t, x)\right| .
\end{aligned}
$$

We note that we only have $\left|Z(t, x)-Z_{\mathrm{s}}(t, x)\right| \lesssim 1$ by (2.11) and (2.13), so that (using the explicit formulas (1.4) and (1.7))

$$
(t+1)^{\frac{\ell+1}{5}}\left|\partial_{z}^{\ell} h_{\mathrm{s}}(t, Z(t, x))-\partial_{z}^{\ell} h_{\mathrm{s}}\left(t, Z_{\mathrm{s}}(t, x)\right)\right| \lesssim(t+1)^{-\frac{1}{5}} \quad \text { for all } x \in[-1,1],
$$


$t \geq 0$, and $\ell \in\{0,1\}$. In view of (2.14), this upgrades to convergence in the original variables

$$
(t+1)^{\frac{\ell+1}{5}}\left|\partial_{z}^{\ell} h(t, z)-\partial_{z}^{\ell} h_{\mathrm{s}}(t, z)\right| \lesssim(t+1)^{-\frac{1}{5}} \quad \text { for all } z \in \mathbb{R}, t \geq 0 \text {, and } \ell \in\{0,1\} \text {. }
$$

The fact that by (2.15) also the (scaled) derivative $(t+1)^{\frac{2}{5}} \partial_{z} h$ converges to $(t+1)^{\frac{2}{5}} \partial_{z} h_{\mathrm{s}}$ in $L^{\infty}(\mathbb{R})$ with the optimal rate $(t+1)^{-\frac{1}{5}}$ is not contained in the previous results $[12,25]$.

Remark 2.6. An analogous reasoning shows that (2.5), (2.6), and (2.11) upgrade to decay in (translated) variables

$$
(t+1)^{\frac{\ell+1}{5}}\left|\partial_{z}^{\ell} h(t, z-\alpha(t))-\partial_{z}^{\ell} h_{\mathrm{s}}(t, z)\right| \lesssim_{\varepsilon}(t+1)^{-1+\varepsilon} \quad \text { for } z \in \mathbb{R}, t \geq 0 \text {, and } \varepsilon>0 \text {. }
$$

Here, $\alpha(t)$ is defined in (2.12) and $\ell \in\{0,1\}$. Equation (2.16) shows the improved decay rate by taking translations of the center of mass $\alpha(t)$ into account, as expected from the linear analysis of Bernoff and Witelski [6], respectively, McCann and Seis $[26]$.

Outline. The rest of the paper will be concerned with the proofs of Theorems 2.1 and 2.2. In section 3 we provide an overview of the most important properties of the weighted Sobolev spaces $\mathcal{H}^{k}$ and their interpolation spaces $\mathcal{H}_{*}^{k}$. We keep the presentation brief and postpone the proofs to Appendix B. In section 4 we derive maximal regularity estimates for the linear degenerate-parabolic problem (1.23). Since $\mathcal{L}$ has a spectral gap, the decay of the solution can be encoded in these estimates. In section 5 we show that the nonlinearity $\mathcal{N}(u)$ (cf. (1.21)) is just a small perturbation of the linear evolution. Thus the corresponding nonlinear problem (1.20) can be treated by a fixed point argument (cf. section 6), proving Theorem 2.1. In section 7 we provide the arguments and proofs for Theorem 2.2, that is, we iteratively prove that the solution $u=u(\tau, x)$ gains regularity for increasing $\tau>0$ through refined estimates of the nonlinearity. Concluding remarks on possible further research are finally presented in section 8 .

3. Embeddings for weighted Sobolev spaces. Most of the proofs of the following results are contained in Appendix B.

$C^{0}$-estimates. Since the nonlinearity $\mathcal{N}(u)$ consists of terms, in which products of up to six terms $\partial_{x}^{j} u(j \in\{0,1,2,3,4\})$ appear (cf. (1.21)), the control of the $C^{0}$ norm of $\partial_{x}^{j} u$ with weights appears to be necessary in order to establish appropriate estimates for the nonlinearity.

Let us start by noting that with the transformation $\tanh s:=x$ one obtains for $u \in$ $C^{\infty}([-1,1])$ and some $\alpha \in \mathbb{R}$ that $\left\|\left(1-x^{2}\right)^{\alpha} u\right\|_{C^{0}([-1,1])}=\|v\|_{L^{\infty}(\mathbb{R})}$, where $v(s):=$ $\left(1-(\tanh s)^{2}\right)^{\alpha} u(\tanh s)$. Then one may use the elementary estimate $\|v\|_{L^{\infty}(\mathbb{R})} \lesssim$ $\|v\|_{H^{1}(\mathbb{R})}$ so that with $\frac{\mathrm{d} x}{\mathrm{~d} s}=1-x^{2}$ we obtain

$$
\begin{aligned}
& \left\|\left(1-x^{2}\right)^{\alpha} u\right\|_{C^{0}([-1,1])}^{2} \\
& \quad \lesssim \int_{-\infty}^{\infty}\left(v^{2}+\left(\partial_{s} v\right)^{2}\right) \mathrm{d} s \\
& \quad=\int_{-1}^{1} \frac{1}{1-x^{2}}\left(\left(1-x^{2}\right)^{2 \alpha} u^{2}+\left(1-x^{2}\right)^{2}\left(\partial_{x}\left(1-x^{2}\right)^{\alpha} u\right)^{2}\right) \mathrm{d} x \\
& \quad \leq \int_{-1}^{1}\left(\left(1+6 \alpha^{2}\right)\left(1-x^{2}\right)^{2 \alpha-1} u^{2}+3\left(1-x^{2}\right)^{2 \alpha+1}\left(\partial_{x} u\right)^{2}\right) \mathrm{d} x,
\end{aligned}
$$


that is,

$$
\left\|\left(1-x^{2}\right)^{\alpha} u\right\|_{C^{0}([-1,1])}^{2} \lesssim_{\alpha} \int_{-1}^{1}\left(\left(1-x^{2}\right)^{2 \alpha-1} u^{2}+\left(1-x^{2}\right)^{2 \alpha+1}\left(\partial_{x} u\right)^{2}\right) \mathrm{d} x .
$$

In fact we can improve estimate (3.1) by applying Hardy's inequality.

Lemma 3.1 (Hardy inequality). Let $\gamma \neq-\frac{1}{2}$ and assume that $w \in H_{\mathrm{loc}}^{1}((0, \infty))$ with $\left\|x^{\gamma+1} \partial_{x} w\right\|_{L^{2}((0, \infty))}<\infty$. Furthermore suppose that there exists a sequence $\left(x_{n}\right)_{n} \subset(0, \infty)$ with $x_{n} \nearrow \infty$ as $n \rightarrow \infty$ if $\gamma>-\frac{1}{2}\left(x_{n} \searrow 0\right.$ as $n \rightarrow \infty$ if $\left.\gamma<-\frac{1}{2}\right)$ such that $w\left(x_{n}\right) \rightarrow 0$ as $n \rightarrow \infty$. Then

$$
\left\|x^{\gamma} w\right\|_{L^{2}((0, \infty))} \leq \frac{2}{|2 \gamma+1|}\left\|x^{\gamma+1} \partial_{x} w\right\|_{L^{2}((0, \infty))} .
$$

We refer to [17, Lemma A.1] or [19] for a proof. A consequence of Lemma 3.1 is the following.

Lemma 3.2. Suppose $\beta>-1$ and $\gamma \in \mathbb{R}$. For every $v \in C^{\infty}([-1,1])$, we have

$$
\int_{-1}^{1}\left(1-x^{2}\right)^{\beta} v^{2} \mathrm{~d} x \lesssim_{\beta, \gamma} \int_{-1}^{1}\left(\left(1-x^{2}\right)^{\gamma} v^{2}+\left(1-x^{2}\right)^{\beta+2}\left(\partial_{x} v\right)^{2}\right) \mathrm{d} x,
$$

where the constant in (3.3) diverges as $\beta \searrow-1$ or $\gamma \nearrow \infty$.

Remark 3.3. We emphasize that an estimate of the form

$$
\int_{-1}^{1}\left(1-x^{2}\right)^{\beta} v^{2} \mathrm{~d} x \lesssim_{\beta} \int_{-1}^{1}\left(1-x^{2}\right)^{\beta+2}\left(\partial_{x} v\right)^{2} \mathrm{~d} x,
$$

which would correspond to the scaling-invariant Hardy inequality of Lemma 3.1, is not true (a nonzero constant is a trivial counterexample).

Lemma 3.2 together with estimate (3.1) can be used to prove the following.

Lemma 3.4. For all $u \in C^{\infty}([-1,1])$, we have

$$
\left\|\left(1-x^{2}\right)^{\alpha} \partial_{x}^{\ell} u\right\|_{C^{0}([-1,1])} \lesssim_{\alpha, k, \ell}|u|_{k+\ell} \quad \text { for } k \geq 1, \ell \geq 0, \alpha \geq \frac{4+\ell-k}{2} \text {, and } \alpha>0 .
$$

Proof. Using the definition of the norms through (1.26), we can replace $u$ by derivatives of $u$. Therefore, it suffices to prove (3.4) for $\ell=0$. This can be obtained by iteratively applying (3.3) to (3.1), i.e.,

$$
\left\|\left(1-x^{2}\right)^{\alpha} u\right\|_{C^{0}([-1,1])}^{2} \lesssim_{k, \alpha} \sum_{j=0}^{k} \int_{-1}^{1}\left(1-x^{2}\right)^{2 \alpha+2 k-1}\left(\partial_{x}^{j} u\right)^{2} \mathrm{~d} x \stackrel{(1.26)}{\leq}|u|_{k}^{2} .
$$

Interpolation spaces. As we noted in the introduction, inequality (3.4) ceases to be true for $k=4+\ell$ and $\alpha=0$ (this is the critical case for which Hardy's inequality is known to fail). This makes the introduction of interpolation spaces necessary (cf. Definition 1.1), for which the estimate is also true in the critical case.

Lemma 3.5. For $\ell \geq 0$ we have

$$
\left\|\partial_{x}^{\ell} u\right\|_{C^{0}([-1,1])} \lesssim_{\ell}|u|_{4+2 \ell, *} \quad \text { for all } u \in \mathcal{H}_{*}^{4+2 \ell} .
$$

The following lemma states that the norm $|\cdot|_{k, *}$ is indeed stronger than $|\cdot|_{k}$ and obeys a standard interpolation estimate. 
Lemma 3.6. For $k \geq 1$ we have

$$
|u|_{k} \lesssim_{k}|u|_{k, *} \lesssim|u|_{k-1}^{\frac{1}{2}}|u|_{k+1}^{\frac{1}{2}} \quad \text { for all } u \in \mathcal{H}^{k+1} .
$$

Additionally, if $0 \leq \ell_{1}<k<\ell_{2}$, then

$$
|u|_{k, *} \lesssim \ell_{2}|u|_{\ell_{1}}^{\frac{\ell_{2}-k}{\ell_{2}-\ell_{1}}}|u|_{\ell_{2}}^{\frac{k-\ell_{1}}{\ell_{2}-\ell_{1}}} \quad \text { for all } u \in \mathcal{H}^{\ell_{2}}
$$

It is quite obvious that (3.8) follows from (3.7) inductively. Combining Lemmas $3.4,3.5$, and 3.6 , we obtain the following.

Corollary 3.7. For all $k \geq 1, \ell \geq 0$, and $\alpha \geq \max \left\{\frac{4+\ell-k}{2}, 0\right\}$, we have

$$
\left\|\left(1-x^{2}\right)^{\alpha} \partial_{x}^{\ell} u\right\|_{C^{0}([-1,1])} \lesssim_{k, \ell}|u|_{k+\ell, *} \quad \text { for all } u \in \mathcal{H}_{*}^{k+\ell} .
$$

Corollary 3.7 turns out to be the basis of all nonlinear estimates in what follows.

We now turn our attention to the discussion of the interpolation norms for our parabolic function spaces: Note that the spaces $C_{\mu}^{0}\left(\bar{I} ; \mathcal{H}^{k}\right)^{*}$ and $C_{\mu}^{0}\left(\bar{I} ; \mathcal{H}_{*}^{k}\right)$, respectively, $L_{\mu}^{2}\left(I ; \mathcal{H}^{k}\right)^{*}$ and $L_{\mu}^{2}\left(I ; \mathcal{H}_{*}^{k}\right)$, are different. Nonetheless, the following inclusions hold true.

Lemma 3.8. For $k \geq 1$ we have

$$
\|u\|_{C_{\mu}^{0}\left(\bar{I} ; \mathcal{H}_{*}^{k}\right)} \lesssim\|u\|_{C_{\mu}^{0}\left(\bar{I} ; \mathcal{H}^{k}\right)^{*}} \quad \text { and } \quad\|u\|_{L_{\mu}^{2}\left(I ; \mathcal{H}_{*}^{k}\right)} \lesssim\|u\|_{L_{\mu}^{2}\left(I ; \mathcal{H}^{k}\right)^{*}}
$$

for all $u \in C_{\mu}^{0}\left(\bar{I} ; \mathcal{H}^{k}\right)^{*}$, respectively, $u \in L_{\mu}^{2}\left(I ; \mathcal{H}^{k}\right)^{*}$.

The proof does not rely on the specific structure of the spaces $\mathcal{H}^{k}$ and can be found in [17, Lemma 1.3].

4. The linear degenerate-parabolic equation. In this section we prove maximal regularity estimates for the linear degenerate-parabolic problem (1.23). Let us introduce the notation

$$
\begin{aligned}
\|u\|_{I} & :=\|u\|_{H_{\mu}^{1}\left(I ; \mathcal{H}^{2}\right)^{*} \cap C_{\mu}^{0}\left(\bar{I} ; \mathcal{H}^{4}\right)^{*} \cap L_{\mu}^{2}\left(I ; \mathcal{H}^{6}\right)^{*}} \\
\left\|u_{0}\right\|_{0} & :=\left\|u_{0}\right\|_{\mathcal{H}_{*}^{4}} \\
\|f\|_{1, I} & :=\|f\|_{L_{\mu}^{2}\left(I ; \mathcal{H}^{2}\right)^{*}}
\end{aligned}
$$

If $I=(0, \infty)$, we will not specify the interval in the notation, i.e., we write $\|\cdot\| \|=$ $\|\cdot \mid\|_{(0, \infty)}$ and $\|\cdot\|_{1}:=\|\cdot\|_{1,(0, \infty)}$ as before.

Proposition 4.1. Suppose $I=\left(0, \tau_{1}\right) \subseteq(0, \infty)$. Then for every $u_{0} \in \mathcal{H}_{*}^{4}$ and every $f \in L^{2}\left(I ; \mathcal{H}^{2}\right)^{*}$, there exists a unique solution

$$
u=\mathcal{T}\left[u_{0}, f\right] \in H^{1}\left(I ; \mathcal{H}^{2}\right)^{*} \cap C^{0}\left(\bar{I} ; \mathcal{H}^{4}\right)^{*} \cap L^{2}\left(I ; \mathcal{H}^{6}\right)^{*} .
$$

This solution obeys the maximal regularity estimate

$$
\|u\|_{I} \lesssim_{\mu}\left\|u_{0}\right\|_{0}+\|f\|_{1, I},
$$

where $\mu<120$.

Proposition 4.1 turns out to be a consequence of a symmetric structure of derivatives of the linear operator $\mathcal{L}$ (cf. (1.19)). 
Lemma 4.2. For each $\ell \in \mathbb{N}_{0}$ we have the commutation relation $\partial_{x}^{\ell} \mathcal{L}=: \mathcal{L}_{\ell} \partial_{x}^{\ell}$, where

$$
\begin{aligned}
\mathcal{L}_{\ell}= & \left(1-x^{2}\right)^{-\ell-3} \partial_{x}^{2}\left(1-x^{2}\right)^{\ell+5} \partial_{x}^{2}-2(\ell+3)(\ell+5)\left(1-x^{2}\right)^{-\ell-3} \partial_{x}\left(1-x^{2}\right)^{\ell+4} \partial_{x} \\
& +(\ell+2)(\ell+3)(\ell+4)(\ell+5) .
\end{aligned}
$$

Proof. One may prove inductively (using (1.19)) that

$$
\begin{aligned}
\mathcal{L}_{\ell}= & \left(1-x^{2}\right)^{2} \partial_{x}^{4}-4(\ell+5) x\left(1-x^{2}\right) \partial_{x}^{3}+2(\ell+4)(\ell+5)\left(3 x^{2}-1\right) \partial_{x}^{2} \\
& +4(\ell+3)(\ell+4)(\ell+5) x \partial_{x}+(\ell+2)(\ell+3)(\ell+4)(\ell+5)
\end{aligned}
$$

and verify in a second step the equivalence of (4.3) and (4.4).

As a consequence we can prove the following.

COROLlary 4.3. The operator $\mathcal{L}$ is symmetric and $\mathcal{L}-\mu$ is coercive with respect to the bilinear forms $\langle\cdot, \cdot\rangle_{\ell}$ for any $\ell \in \mathbb{N}_{0}$ and $\eta:=(\ell+2) \cdots(\ell+5)-\mu>0$, i.e.,

$$
\begin{aligned}
\langle u, \mathcal{L} v\rangle_{\ell} & =\langle\mathcal{L} u, v\rangle_{\ell}, \\
\langle u,(\mathcal{L}-\mu) u\rangle_{\ell} & \sim_{\eta}[u]_{\ell, \ell+2}^{2} \quad \text { for all } u, v \in C^{\infty}([-1,1]) . \\
{[(\mathcal{L}-\mu) u]_{\ell} } & \sim_{\eta}[u]_{\ell, \ell+4} .
\end{aligned}
$$

Proof. Using Lemma 4.2 and integration by parts, we obtain for $u, v \in C^{\infty}([-1,1])$

$$
\begin{aligned}
\langle u, \mathcal{L} v\rangle_{\ell}= & \int_{-1}^{1}\left(1-x^{2}\right)^{\ell+5}\left(\partial_{x}^{\ell+2} u\right)\left(\partial_{x}^{\ell+2} v\right) \mathrm{d} x \\
& +2(\ell+3)(\ell+5) \int_{-1}^{1}\left(1-x^{2}\right)^{\ell+4}\left(\partial_{x}^{\ell+1} u\right)\left(\partial_{x}^{\ell+1} v\right) \mathrm{d} x \\
& +(\ell+2) \cdots(\ell+5) \int_{-1}^{1}\left(1-x^{2}\right)^{\ell+3}\left(\partial_{x}^{\ell} u\right)\left(\partial_{x}^{\ell} v\right) \mathrm{d} x \\
= & \langle u, v\rangle_{\ell+2}+2(\ell+3)(\ell+5)\langle u, v\rangle_{\ell+1}+(\ell+2) \cdots(\ell+5)\langle u, v\rangle_{\ell},
\end{aligned}
$$

from which the claim immediately follows.

Proof of Proposition 4.1. We only provide heuristics here and prove estimate (4.2) on a time-continuum level. The subsequent arguments can be made rigorous using abstract semigroup theory (cf. $[24,28,31])$ : In fact, since by Corollary 4.3 the operator $(\mathcal{L}-\mu): \mathcal{H}^{k} \supset \mathcal{H}^{k+4} \rightarrow \mathcal{H}^{k}$ for $\mu<120$ is symmetric and coercive, abstract theory (cf. [28, Proposition 4.2]) right away yields existence and uniqueness and the maximal regularity estimate (4.13) (see below).

By the definition of our Sobolev spaces $\mathcal{H}^{k}$ (cf. (1.27)) and a standard approximation argument in time, we can assume that $u$ is smooth.

First, we multiply (1.23a) with an exponential weight $e^{\mu \tau}$ and obtain for $v:=e^{\mu \tau} u$ and $g:=e^{\mu \tau} f$ the equation

$$
\partial_{\tau} v+(\mathcal{L}-\mu) v=g \text { for } \tau \in I \text { and }-1<x<1 .
$$

Now we test $(4.6)$ with $(\mathcal{L}-\mu) v$ with respect to $(\cdot, \cdot)_{k}$ and obtain

$$
\left(\partial_{\tau} v,(\mathcal{L}-\mu) v\right)_{k}+|(\mathcal{L}-\mu) v|_{k}^{2}=(g,(\mathcal{L}-\mu) v)_{k} \quad \text { for } \tau \in I .
$$


For the right-hand side of (4.7) we use Young's inequality and so obtain

$$
\left|(g,(\mathcal{L}-\mu) v)_{k}\right| \leq \frac{1}{2}|g|_{k}^{2}+\frac{1}{2}|(\mathcal{L}-\mu) v|_{k}^{2} .
$$

Equation (4.8) in (4.7) yields

$$
2\left(\partial_{\tau} v,(\mathcal{L}-\mu) v\right)_{k}+|(\mathcal{L}-\mu) v|_{k}^{2} \leq|g|_{k}^{2} \quad \text { for } \tau \in I .
$$

For the first term of (4.9), we observe that by Corollary 4.3

$$
\left(\partial_{\tau} v,(\mathcal{L}-\mu) v\right)_{k} \stackrel{(4.5)}{=} \frac{1}{2}\left(\partial_{\tau} v,(\mathcal{L}-\mu) v\right)_{k}+\frac{1}{2}\left(\partial_{\tau}(\mathcal{L}-\mu) v, v\right)_{k}=\frac{1}{2} \frac{\mathrm{d}}{\mathrm{d} \tau}(v,(\mathcal{L}-\mu) v)_{k} .
$$

We may use (4.10) in (4.9) and thus get

$$
\frac{\mathrm{d}}{\mathrm{d} \tau}(v,(\mathcal{L}-\mu) v)_{k}+|(\mathcal{L}-\mu) v|_{k}^{2} \leq|g|_{k}^{2} .
$$

Integrating (4.11) in $\tau$ and using Corollary 4.3 yields

$$
\sup _{\tau \in I}|v(\tau)|_{k+2}^{2}+\int_{I}|v(\tau)|_{k+4}^{2} \mathrm{~d} \tau \lesssim \mu\left|u_{0}\right|_{k+2}^{2}+\int_{I}|g(\tau)|_{k}^{2} \mathrm{~d} \tau
$$

for $\mu<120$. Undoing the transformations $v=e^{\mu \tau} u$ and $g=e^{\mu \tau} f$ and using (1.23a) for getting control on $\partial_{\tau} u$, we obtain

$\sup _{\tau \in I} e^{2 \mu \tau}|u(\tau)|_{k+2}^{2}+\int_{I} e^{2 \mu \tau}\left(\left|\partial_{\tau} u(\tau)\right|_{k}^{2}+|u(\tau)|_{k+4}^{2}\right) \mathrm{d} \tau \lesssim \mu\left|u_{0}\right|_{k+2}^{2}+\int_{I} e^{2 \mu \tau}|f(\tau)|_{k}^{2} \mathrm{~d} \tau$ for $\mu<120$. Interpolating (4.13) for $k=1$ and $k=3$, we immediately arrive at $(4.2)$.

5. The nonlinearity. For the proof of Theorem 2.1, we establish appropriate estimates for the nonlinearity. We start with a basic observation on the interpolation norm defined in (1.29a).

LEMma 5.1. For all $\mu \geq 0$ we have

$$
\begin{aligned}
\|v\|_{L_{\mu}^{2}\left(I ; \mathcal{H}^{2}\right)^{*}} \lesssim & \|v\|_{L_{\mu}^{2}\left(I ; \mathcal{H}^{0}\right)} \\
& +\int_{0}^{\infty} \inf _{v=v_{-}+v_{+}}\left(\int_{0}^{\infty} e^{-2 \mu \tau}\left(\sigma^{-1}\left[v_{-}\right]_{1}^{2}+\sigma\left[v_{+}\right]_{1,3}^{2}\right) \mathrm{d} \tau\right)^{\frac{1}{2}} \frac{\mathrm{d} \sigma}{\sigma}
\end{aligned}
$$

for all $v \in L_{\mu}^{2}\left(I ; \mathcal{H}^{2}\right)^{*}$.

In the interpolation seminorm in (5.1) at least one derivative acts on $v_{-}$, respectively, $v_{+}$. This will turn out to be convenient for estimating the nonlinearity $\mathcal{N}(u)$.

Proof of Lemma 5.1. For $k \geq 0$ we define the spaces $\mathcal{H}_{+}^{k}$ as the completion of all $v \in C^{\infty}([-1,1])$ with respect to $|\cdot|_{k,+}$, where

$$
|v|_{k,+}^{2}:=\sum_{j=0}^{k} \int_{-1}^{1}\left(1-x^{2}\right)^{j+4}\left(\partial_{x}^{j} v\right)^{2} \mathrm{~d} x .
$$


Apparently

$$
\left|\partial_{x} v\right|_{k,+} \stackrel{(1.25)}{=}[v]_{1, k+1},
$$

which we will use in what follows. Furthermore, we define the linear operation $T$ through $T v(x):=\int_{0}^{x} v\left(x^{\prime}\right) \mathrm{d} x^{\prime}$. Hence almost everywhere $\partial_{x} T v(x)=v(x)$. We claim that $T: \mathcal{H}_{+}^{k} \rightarrow \mathcal{H}^{k+1}$ is bounded. Indeed we have

$$
[T v]_{j+1}^{2}=\int_{-1}^{1}\left(1-x^{2}\right)^{j+4}\left(\partial_{x}^{j} v\right)^{2} \mathrm{~d} x \leq|v|_{k,+}^{2} \quad \text { for all } j=0, \ldots, k .
$$

Additionally

$$
\begin{aligned}
|T v|_{0}^{2} & =\int_{-1}^{1}\left(1-x^{2}\right)^{3}\left(\int_{0}^{x} v\left(x^{\prime}\right) \mathrm{d} x^{\prime}\right)^{2} \mathrm{~d} x \\
& \leq \int_{-1}^{1}\left(1-x^{2}\right)^{-\frac{1}{2}}\left(\int_{0}^{x}\left(1-\left(x^{\prime}\right)^{2}\right)^{\frac{7}{4}}\left|v\left(x^{\prime}\right)\right| \mathrm{d} x^{\prime}\right)^{2} \mathrm{~d} x \lesssim\left(\int_{-1}^{1}\left(1-x^{2}\right)^{\frac{7}{4}}|v| \mathrm{d} x\right)^{2} \\
& \leq\left(\int_{-1}^{1}\left(1-x^{2}\right)^{-\frac{1}{2}} \mathrm{~d} x\right) \times\left(\int_{-1}^{1}\left(1-x^{2}\right)^{4} v^{2} \mathrm{~d} x\right) \lesssim|v|_{0,+}^{2} .
\end{aligned}
$$

Setting $T v(\tau, x):=(T v(\tau, \cdot))(x)$, also $T: L_{\mu}^{2}\left(I ; \mathcal{H}_{+}^{k}\right) \rightarrow L_{\mu}^{2}\left(I ; \mathcal{H}^{k+1}\right)$ is bounded. By interpolation, we infer that also

$$
T:\left(L_{\mu}^{2}\left(I ; \mathcal{H}_{+}^{0}\right), L_{\mu}^{2}\left(I ; \mathcal{H}_{+}^{2}\right)\right)_{\frac{1}{2}, 1} \rightarrow L_{\mu}^{2}\left(I ; \mathcal{H}^{2}\right)^{*}
$$

is bounded, that is,

$$
\|T v\|_{L_{\mu}^{2}\left(I ; \mathcal{H}^{2}\right)^{*}} \lesssim\|v\|_{\left(L_{\mu}^{2}\left(I ; \mathcal{H}_{+}^{0}\right), L_{\mu}^{2}\left(I ; \mathcal{H}_{+}^{2}\right)\right)_{\frac{1}{2}, 1}} .
$$

Replacing $v$ by $\partial_{x} v$ and noting that $\left(T \partial_{x} v\right)(\tau, x)=v(\tau, x)-v(\tau, 0)$ almost everywhere, we infer

$$
\left\|v-v_{\mid x=0}\right\|_{L_{\mu}^{2}\left(I ; \mathcal{H}^{2}\right)^{*}} \lesssim\left\|\partial_{x} v\right\|_{\left(L_{\mu}^{2}\left(I ; \mathcal{H}_{+}^{0}\right), L_{\mu}^{2}\left(I ; \mathcal{H}_{+}^{2}\right)\right)_{\frac{1}{2}, 1}} .
$$

By the triangle inequality and a standard embedding

$$
\begin{aligned}
\|v\|_{L_{\mu}^{2}\left(I ; \mathcal{H}^{2}\right)^{*}} & \leq\left\|v_{\mid x=0}\right\|_{L_{\mu}^{2}(I)}+\left\|v-v_{\mid x=0}\right\|_{L_{\mu}^{2}\left(I ; \mathcal{H}^{2}\right)^{*}} \\
& \stackrel{(5.3)}{\lesssim}\left\|v_{\mid x=0}\right\|_{L_{\mu}^{2}(I)}+\left\|\partial_{x} v\right\|_{\left(L_{\mu}^{2}\left(I ; \mathcal{H}_{+}^{0}\right), L_{\mu}^{2}\left(I ; \mathcal{H}_{+}^{2}\right)\right)_{\frac{1}{2}, 1}} \\
& \lesssim\|v\|_{L_{\mu}^{2}\left(I ; H^{1}\left(\left(-\frac{1}{2}, \frac{1}{2}\right)\right)\right)}+\left\|\partial_{x} v\right\|_{\left(L_{\mu}^{2}\left(I ; \mathcal{H}_{+}^{0}\right), L_{\mu}^{2}\left(I ; \mathcal{H}_{+}^{2}\right)\right)_{\frac{1}{2}, 1}} \\
& \lesssim\|v\|_{L_{\mu}^{2}\left(I ; \mathcal{H}^{0}\right)}+\left\|\partial_{x} v\right\|_{\left(L_{\mu}^{2}\left(I ; \mathcal{H}_{+}^{0}\right), L_{\mu}^{2}\left(I ; \mathcal{H}_{+}^{2}\right)\right)_{\frac{1}{2}, 1} .}
\end{aligned}
$$

Using (5.2), (5.4) yields (5.1).

Lemma 5.1 can be used to prove the main estimate for the nonlinearity.

Proposition 5.2. For any interval $I \subseteq(0, \infty)$ we have

$$
\|\mathcal{N}(u)\|_{1, I} \lesssim \max _{m=2,6}\|u\|_{I}^{m}
$$


and

$$
\left\|\mathcal{N}\left(u_{1}\right)-\mathcal{N}\left(u_{2}\right)\right\|_{1, I} \lesssim \max _{m=1,5}\left(\left\|u_{1}\right\|_{I}^{m}+\left\|u_{2}\right\|_{I}^{m}\right)\left\|u_{1}-u_{2}\right\|_{I}
$$

for any $\mu \geq 0$ and

$$
u, u_{1}, u_{2} \in H_{\mu}^{1}\left(I ; \mathcal{H}^{2}\right)^{*} \cap C_{\mu}^{0}\left(\bar{I} ; \mathcal{H}^{4}\right)^{*} \cap L_{\mu}^{2}\left(I ; \mathcal{H}^{6}\right)^{*} .
$$

Proof. It suffices to prove (5.5b), since (5.5a) follows from (5.5b) by setting $u_{1}:=u$ and $u_{2}:=0$. For estimating $\mathcal{N}\left(u_{1}\right)-\mathcal{N}\left(u_{2}\right)$ in the interpolation norm $\|\cdot\|_{L_{\mu}^{2}\left(I ; \mathcal{H}^{2}\right)^{*}}=\|\cdot\|_{1, I}$, we use estimate (5.1) of Lemma 5.1:

$$
\begin{aligned}
& \left\|\mathcal{N}\left(u_{1}\right)-\mathcal{N}\left(u_{2}\right)\right\|_{L^{2}\left(I ; \mathcal{H}^{2}\right)^{*}} \\
& \quad \lesssim\left\|\mathcal{N}\left(u_{1}\right)-\mathcal{N}\left(u_{2}\right)\right\|_{L^{2}\left(I ; \mathcal{H}^{0}\right)} \\
& \quad+\int_{0}^{\infty} \inf _{\mathcal{N}\left(u_{1}\right)-\mathcal{N}\left(u_{2}\right)=N_{-}+N_{+}}\left(\int_{0}^{\infty} e^{2 \mu \tau}\left(\sigma^{-1}\left[N_{-}\right]_{1}^{2}+\sigma\left[N_{+}\right]_{1,3}^{2}\right) \mathrm{d} \tau\right)^{\frac{1}{2}} \frac{\mathrm{d} \sigma}{\sigma} .
\end{aligned}
$$

We treat the second and third lines in (5.6) separately.

Estimate of $\left\|\mathcal{N}\left(u_{1}\right)-\mathcal{N}\left(u_{2}\right)\right\|_{L^{2}\left(I ; \mathcal{H}^{0}\right)}$. Our aim is to show that

$$
\left\|\mathcal{N}\left(u_{1}\right)-\mathcal{N}\left(u_{2}\right)\right\|_{L_{\mu}^{2}\left(I ; \mathcal{H}^{0}\right)} \lesssim \max _{m=1,5}\left(\left\|u_{1}\right\|_{I}^{m}+\left\|u_{2}\right\|_{I}^{m}\right)\left\|u_{1}-u_{2}\right\|_{I} .
$$

Therefore we use (1.21), that is, $\mathcal{N}(u)=\sum_{m=2}^{6} \mathcal{M}_{m}(u, \ldots, u)$ with $m \in\{2, \ldots, 6\}$, where $\mathcal{M}_{m}$ is an $m$-linear form being a linear combination of terms of the form

$$
\partial_{x}^{\ell_{0}}\left(1-x^{2}\right)^{2} \times \prod_{j=1}^{m} \partial_{x}^{\ell_{j}} v_{j} \quad \text { with } \sum_{j=0}^{m} \ell_{j}=: \ell=4 \text { and } \ell_{1} \geq \ell_{2} \geq \cdots \geq \ell_{m} .
$$

Since

$$
\begin{aligned}
\mathcal{N}\left(u_{1}\right)-\mathcal{N}\left(u_{2}\right) & =\sum_{m=2}^{6}\left(\mathcal{M}_{m}\left(u_{1}, \ldots, u_{1}\right)-\mathcal{M}_{m}\left(u_{2}, \ldots, u_{2}\right)\right) \\
& =\sum_{m=2}^{6}\left(\mathcal{M}_{m}\left(u_{1}-u_{2}, u_{1}, \ldots, u_{1}\right)+\cdots+\mathcal{M}_{m}\left(u_{2}, \ldots, u_{2}, u_{1}-u_{2}\right)\right),
\end{aligned}
$$

it suffices to estimate terms of the form

$$
\int_{-1}^{1}\left(1-x^{2}\right)^{3}\left(\partial_{x}^{\ell_{0}}\left(1-x^{2}\right)^{2} \prod_{j=1}^{m} \partial_{x}^{\ell_{j}} v_{j}\right)^{2} \mathrm{~d} x, \text { where } m \in\{2, \ldots, 6\}, \quad \sum_{j=0}^{m} \ell_{j}=: \ell=4,
$$

$\ell_{1} \geq \cdots \geq \ell_{m}, v_{j} \in\left\{u_{1}, u_{2}, u_{1}-u_{2}\right\}$, and $v_{j}=u_{1}-u_{2}$ for exactly one $j \in\{1, \ldots, m\}$. By applying Lemma $3.2 \ell_{0}$-times, we may assume without loss of generality $\ell_{0}=0$ 
and $\ell \in\{0, \ldots, 4\}$. By the ordering necessarily $\ell_{1} \geq 1$ or $\ell=0$. Then we may estimate (5.10) as follows:

$$
\begin{aligned}
& \int_{-1}^{1}\left(1-x^{2}\right)^{7}\left(\prod_{j=1}^{m} \partial_{x}^{\ell_{j}} v_{j}\right)^{2} \mathrm{~d} x \\
& \quad \lesssim \prod_{j=2}^{m}\left\|\left(1-x^{2}\right)^{\ell_{j}} \partial_{x}^{\ell_{j}} v_{j}\right\|_{C^{0}([-1,1])}^{2} \times \int_{-1}^{1}\left(1-x^{2}\right)^{7+2 \ell_{1}-2 \ell}\left(\partial_{x}^{\ell_{1}} v_{1}\right)^{2} \mathrm{~d} x .
\end{aligned}
$$

For the second term, we apply Lemma 3.2 iteratively and obtain

$$
\int_{-1}^{1}\left(1-x^{2}\right)^{7+2 \ell_{1}-2 \ell}\left(\partial_{x}^{\ell_{1}} v_{1}\right)^{2} \mathrm{~d} x \lesssim \sum_{j=\ell_{1}}^{\ell} \int_{-1}^{1}\left(1-x^{2}\right)^{7}\left(\partial_{x}^{j} v_{1}\right)^{2} \mathrm{~d} x \lesssim\left|v_{1}\right|_{4}^{2} .
$$

Since $\ell_{j} \leq 2$ for $j \geq 2$, we can use Corollary 3.7 for the other terms and end up with

$$
\int_{-1}^{1}\left(1-x^{2}\right)^{7}\left(\prod_{j=1}^{m} \partial_{x}^{\ell_{j}} v_{j}\right)^{2} \mathrm{~d} x \lesssim\left|v_{1}\right|_{4}^{2} \times \prod_{j=2}^{m}\left|v_{j}\right|_{4, *}^{2},
$$

which in view of (5.8), (5.9), and (5.10) upgrades to

$$
\begin{aligned}
\left|\mathcal{N}\left(u_{1}\right)-\mathcal{N}\left(u_{2}\right)\right|_{0} \lesssim & \max _{m=1,5}\left(\left|u_{1}\right|_{4, *}^{m}+\left|u_{2}\right|_{4, *}^{m}\right) \times\left|u_{1}-u_{2}\right|_{4} \\
& +\max _{m=0,4}\left(\left|u_{1}\right|_{4, *}^{m}+\left|u_{2}\right|_{4, *}^{m}\right) \times\left(\left|u_{1}\right|_{4}+\left|u_{2}\right|_{4}\right) \times\left|u_{1}-u_{2}\right|_{4, *}
\end{aligned}
$$

Integrating this expression in time and using that $\mu \geq 0$ as well as Lemma 3.8, we obtain

$$
\begin{aligned}
& \left\|\mathcal{N}\left(u_{1}\right)-\mathcal{N}\left(u_{2}\right)\right\|_{L_{\mu}^{2}\left(I ; \mathcal{H}^{0}\right)} \\
& \lesssim \max _{m=1,5}\left(\left\|u_{1}\right\|_{C_{\mu}^{0}\left(I ; \mathcal{H}^{4}\right)^{*}}^{m}+\left\|u_{2}\right\|_{C_{\mu}^{0}\left(I ; \mathcal{H}^{4}\right)^{*}}^{m}\right) \times\left\|u_{1}-u_{2}\right\|_{L_{\mu}^{2}\left(I ; \mathcal{H}^{4}\right)} \\
& \quad+\max _{m=0,4}\left(\left\|u_{1}\right\|_{C_{\mu}^{0}\left(I ; \mathcal{H}^{4}\right)^{*}}^{m}+\left\|u_{2}\right\|_{C_{\mu}^{0}\left(I ; \mathcal{H}^{4}\right)^{*}}^{m}\right) \times\left(\left\|u_{1}\right\|_{L_{\mu}^{2}\left(I ; \mathcal{H}^{4}\right)}+\left\|u_{2}\right\|_{L_{\mu}^{2}\left(I ; \mathcal{H}^{4}\right)}\right) \\
& \quad \times\left\|u_{1}-u_{2}\right\|_{C_{\mu}^{0}\left(I ; \mathcal{H}^{4}\right)^{*}}
\end{aligned}
$$

which, in view of the definitions of the parabolic norms in (4.1), yields (5.7).

Estimate of the interpolation seminorm in (5.6). We begin with some observations on the structure of the nonlinearity. Note that in view of (1.21), the derivative $\partial_{x} \mathcal{N}(u)$ is a sum of terms of the form

$$
\alpha\left(m, \ell_{0}, \ldots, \ell_{m}\right) \partial_{x}^{\ell_{0}}\left(1-x^{2}\right)^{2} \times \prod_{j=1}^{m} \partial_{x}^{\ell_{j}} u \quad \text { with } \alpha \in \mathbb{R}, \quad \sum_{j=0}^{m} \ell_{j}=: \ell=5,
$$

and $m \in\{2, \ldots, 6\}$. For convenience we assume $\ell_{1} \geq \ell_{2} \geq \cdots \geq \ell_{m}$ and we may also assume $\ell_{0} \leq 4$ here and in what follows, as otherwise the term (5.11) vanishes. 
Therefore also $\ell_{1} \geq 1$, which will turn out to be beneficial later on. Then we note that we can write

$$
\partial_{x} \mathcal{N}(u)=\sum_{m=2}^{6} \tilde{\mathcal{M}}_{m}(u, \ldots, u)
$$

where $\tilde{\mathcal{M}}_{m}\left(v_{1}, \ldots, v_{m}\right)$ is an $m$-linear form being the sum of all terms

$$
\alpha\left(m, \ell_{0}, \ldots, \ell_{m}\right) \partial_{x}^{\ell_{0}}\left(1-x^{2}\right)^{2} \times \prod_{j=1}^{m} \partial_{x}^{\ell_{j}} v_{j} \quad \text { with } \sum_{j=0}^{m} \ell_{j}=: \ell=5,
$$

and where $\ell_{1} \geq \cdots \geq \ell_{m}$. Furthermore, we can replace $\tilde{\mathcal{M}}_{m}$ by $\mathcal{M}_{m}^{\prime}=\tilde{\mathcal{M}}_{m}+\hat{\mathcal{M}}_{m}$ in (5.12), where $\hat{\mathcal{M}}_{m}$ is any $m$-linear form $\hat{\mathcal{M}}_{m}=\hat{\mathcal{M}}_{m}\left(v_{1}, \ldots, v_{m}\right)$ with

$$
\hat{\mathcal{M}}_{m}\left(v_{1}, \ldots, v_{m}\right) \equiv 0 \quad \text { if } v_{1} \equiv v_{2} \cdots \equiv v_{m} .
$$

It seems necessary to introduce $\hat{\mathcal{M}}_{m}$ in order to be able to deal with terms in which only one derivative acts on $v_{1}$ in $\partial_{x}^{2} \mathcal{M}_{m}^{\prime}\left(v_{1}, \ldots, v_{m}\right)$.

We will choose $\hat{\mathcal{M}}_{m}$ iteratively as the sum of $\hat{\mathcal{M}}_{m}^{(n)}$ with $n=1,2,3$. First we define $\hat{\mathcal{M}}_{m}^{(1)}$ as the sum of all

$$
\begin{aligned}
& \alpha m^{\prime} \int_{0}^{x} \partial_{x}^{\ell_{0}}\left(1-x_{1}^{2}\right)^{2} \partial_{x}^{2} v_{1} \prod_{j=2}^{m^{\prime}} \partial_{x} v_{j} \prod_{j=m^{\prime}+1}^{m} v_{j} \mathrm{~d} x_{1} \\
& -\alpha \sum_{i=1}^{m^{\prime}} \int_{0}^{x} \partial_{x}^{\ell_{0}}\left(1-x_{1}^{2}\right)^{2} \partial_{x}^{2} v_{i} \prod_{\substack{j=1 \\
j \neq i}}^{m^{\prime}} \partial_{x} v_{j} \prod_{j=m^{\prime}+1}^{m} v_{j} \mathrm{~d} x_{1},
\end{aligned}
$$

where $\alpha=\alpha\left(m, \ell_{0}, 1, \ldots, 1,0, \ldots, 0\right), \ell_{j}=1$ for $1 \leq j \leq m^{\prime}$, and $\ell_{j}=0$ for $j \geq m^{\prime}+1$, with $m^{\prime}=5-\ell_{0}$. By this choice, $\partial_{x}\left(\tilde{\mathcal{M}}_{m}\left(v_{1}, \ldots, v_{m}\right)+\hat{\mathcal{M}}_{m}^{(1)}\left(v_{1}, \ldots, v_{m}\right)\right)$ is a sum of terms of the form

$$
\beta\left(m, \ell_{0}, \ldots, \ell_{m}\right) \partial_{x}^{\ell_{0}}\left(1-x^{2}\right)^{2} \times \prod_{j=1}^{m} \partial_{x}^{\ell_{j}} v_{j} \quad \text { with } \sum_{j=0}^{m} \ell_{j}=: \ell=6,
$$

$\beta \in \mathbb{R}$, and where $\ell_{j} \leq 1$ for $j \geq 2$ provided $\ell_{1}=1$. By renumbering without loss of generality $\ell_{j}=1$ for $1 \leq j \leq m^{\prime}$ and $\ell_{j}=0$ for $j \geq m^{\prime}+1$ if $\ell_{1}=1$ (here $m^{\prime}=6-\ell_{0}$ ). Then we may define $\hat{\mathcal{M}}_{m}^{(2)}\left(v_{1}, \ldots, v_{m}\right)$ as the sum of all

$$
\begin{aligned}
& \beta m^{\prime} \int_{0}^{x} \int_{0}^{x_{1}} \partial_{x}^{\ell_{0}}\left(1-x_{2}^{2}\right)^{2} \partial_{x}^{2} v_{1} \prod_{j=2}^{m^{\prime}} \partial_{x} v_{j} \prod_{j=m^{\prime}+1}^{m} v_{j} \mathrm{~d} x_{2} \mathrm{~d} x_{1} \\
& -\beta \sum_{i=1}^{m^{\prime}} \int_{0}^{x} \int_{0}^{x_{1}} \partial_{x}^{\ell_{0}}\left(1-x_{2}^{2}\right)^{2} \partial_{x}^{2} v_{i} \prod_{\substack{j=1 \\
j \neq i}}^{m^{\prime}} \partial_{x} v_{j} \prod_{j=m^{\prime}+1}^{m} v_{j} \mathrm{~d} x_{2} \mathrm{~d} x_{1},
\end{aligned}
$$

where $\ell_{1}=1$. This ensures that

$$
\partial_{x}^{2}\left(\tilde{\mathcal{M}}_{m}\left(v_{1}, \ldots, v_{m}\right)+\hat{\mathcal{M}}_{m}^{(1)}\left(v_{1}, \ldots, v_{m}\right)+\hat{\mathcal{M}}_{m}^{(2)}\left(v_{1}, \ldots, v_{m}\right)\right)
$$


is a sum of terms of the form

$$
\gamma\left(m, \ell_{0}, \ldots, \ell_{m}\right) \partial_{x}^{\ell_{0}}\left(1-x^{2}\right)^{2} \times \prod_{j=1}^{m} \partial_{x}^{\ell_{j}} v_{j} \quad \text { with } \sum_{j=0}^{m} \ell_{j}=: \ell=7,
$$

$\gamma \in \mathbb{R}$, and where $\ell_{j} \leq 1$ for $j \geq 2$ provided $\ell_{1}=1$. Again by renumbering without loss of generality $\ell_{j}=1$ for $1 \leq j \leq m^{\prime}$ and $\ell_{j}=0$ for $j \geq m^{\prime}+1$ (with $m^{\prime}=7-\ell_{0}$ ) in those cases where $\ell_{1}=1$. Then we define $\hat{\mathcal{M}}_{m}^{(3)}\left(v_{1}, \ldots, v_{m}\right)$ as the sum of all

$$
\begin{aligned}
& \gamma m^{\prime} \int_{0}^{x} \int_{0}^{x_{1}} \partial_{x}^{\ell_{0}}\left(1-x_{2}^{2}\right)^{2} \prod_{j=m^{\prime}+1}^{m} v_{j} \int_{0}^{x_{2}} \partial_{x}^{2} v_{1} \prod_{j=2}^{m^{\prime}} \partial_{x} v_{j} \mathrm{~d} x_{3} \mathrm{~d} x_{2} \mathrm{~d} x_{1} \\
& -\gamma \sum_{i=1}^{m^{\prime}} \int_{0}^{x} \int_{0}^{x_{1}} \partial_{x}^{\ell_{0}}\left(1-x_{2}^{2}\right)^{2} \prod_{j=m^{\prime}+1}^{m} v_{j} \int_{0}^{x_{2}} \partial_{x}^{2} v_{i} \prod_{\substack{j=1 \\
j \neq i}}^{m^{\prime}} \partial_{x} v_{j} \mathrm{~d} x_{3} \mathrm{~d} x_{2} \mathrm{~d} x_{1},
\end{aligned}
$$

where $\ell_{1}=1$.

In view of (5.12) with $\tilde{\mathcal{M}}_{m}$ replaced by $\mathcal{M}_{m}^{\prime}$, we have the identification

$$
\begin{aligned}
\partial_{x} \mathcal{N}\left(u_{1}\right)-\partial_{x} \mathcal{N}\left(u_{2}\right) \\
\quad=\sum_{m=2}^{6}\left(\mathcal{M}_{m}^{\prime}\left(u_{1}-u_{2}, u_{1}, \ldots, u_{1}\right)+\cdots+\mathcal{M}_{m}^{\prime}\left(u_{2}, \ldots, u_{2}, u_{1}-u_{2}\right)\right) .
\end{aligned}
$$

For each term $\mathcal{M}_{m}^{\prime}\left(v_{1}, \ldots, v_{m}\right)$ appearing in (5.19) we may decompose

$\mathcal{M}_{m}^{\prime}\left(v_{1}, \ldots, v_{m}\right)=\mathcal{M}_{m}^{\prime}\left(v_{-}, v_{2}, \ldots, v_{m}\right)+\mathcal{M}_{m}^{\prime}\left(v_{+}, v_{2}, \ldots, v_{m}\right)$ where $v_{1}=v_{-}+v_{+}$.

Using the notation $|v|_{k,+}^{2}:=\sum_{j=0}^{k} \int_{-1}^{1}\left(1-x^{2}\right)^{j+4}\left(\partial_{x}^{j} v\right)^{2} \mathrm{~d} x$, the interpolation term in (5.6) can be bound by the sum of all terms of the form

$$
\begin{aligned}
\int_{0}^{\infty} \inf _{v_{1}=v_{-}+v_{+}}\left(\int _ { I } e ^ { 2 \mu \tau } \left(\sigma^{-1}\left|\mathcal{M}_{m}^{\prime}\left(v_{-}, v_{2}, \ldots, v_{m}\right)\right|_{0,+}^{2}\right.\right. \\
\left.\left.+\sigma\left|\mathcal{M}_{m}^{\prime}\left(v_{+}, v_{2}, \ldots, v_{m}\right)\right|_{2,+}^{2}\right) \mathrm{~d} \tau\right)^{\frac{1}{2}} \frac{\mathrm{d} \sigma}{\sigma},
\end{aligned}
$$

where $m \in\{2, \ldots, 6\}, v_{j} \in\left\{u_{1}, u_{2}, u_{1}-u_{2}\right\}$, and $v_{j}=u_{1}-u_{2}$ for exactly one $j \in\{1, \ldots, m\}$. We will treat the norms

$$
\left|\mathcal{M}_{m}^{\prime}\left(v_{-}, v_{2}, \ldots, v_{m}\right)\right|_{0,+} \quad \text { and } \quad\left|\mathcal{M}_{m}^{\prime}\left(v_{+}, v_{2}, \ldots, v_{m}\right)\right|_{2,+}
$$

separately.

Estimate of $\left|\mathcal{M}_{m}^{\prime}\left(v_{-}, v_{2}, \ldots, v_{m}\right)\right|_{0,+}$. We claim

$$
\left|\mathcal{M}_{m}^{\prime}\left(v_{-}, v_{2}, \ldots, v_{m}\right)\right|_{0,+} \lesssim \prod_{j=2}^{m}\left|v_{j}\right|_{4, *} \times\left|v_{-}\right|_{5} \quad \text { for } m \in\{2, \ldots, 6\} .
$$

We start by estimating $\left|\tilde{\mathcal{M}}_{m}\left(v_{-}, v_{2}, \ldots, v_{m}\right)\right|_{0,+}$ for $m \in\{2, \ldots, 6\}$. In view of (5.13), we have to estimate terms of the form

$$
\left(1-x^{2}\right)^{4-\ell_{0}} \times \partial_{x}^{\ell_{1}} v_{-} \times \prod_{j=2}^{m} \partial_{x}^{\ell_{j}} v_{j} \quad \text { with } \ell:=\sum_{j=0}^{m} \ell_{j}=5
$$


in $L^{2}((-1,1))$, where $m \in\{2, \ldots, 6\}, \ell_{0} \leq 4, \ell_{1} \geq \cdots \geq \ell_{m}$, and hence necessarily $\ell_{1} \geq 1$. This can be easily achieved by noting that

$$
\begin{aligned}
& \int_{-1}^{1}\left(1-x^{2}\right)^{8-2 \ell_{0}}\left(\partial_{x}^{\ell_{1}} v_{-}\right)^{2} \prod_{j=2}^{m}\left(\partial_{x}^{\ell_{j}} v_{j}\right)^{2} \mathrm{~d} x \\
& \leq \prod_{j=2}^{m}\left\|\left(1-x^{2}\right)^{\ell_{j}} \partial_{x}^{\ell_{j}} v_{j}\right\|_{C^{0}([-1,1])}^{2} \times \int_{-1}^{1}\left(1-x^{2}\right)^{8+2 \ell_{1}-2 \ell}\left(\partial_{x}^{\ell_{1}} v_{-}\right)^{2} \mathrm{~d} x \\
& \quad \lesssim \prod_{j=2}^{m}\left|v_{j}\right|_{4, *}^{2} \times \sum_{j=\ell_{1}}^{\ell} \int_{-1}^{1}\left(1-x^{2}\right)^{8}\left(\partial_{x}^{j} v_{-}\right)^{2} \mathrm{~d} x \leq \prod_{j=2}^{m}\left|v_{j}\right|_{4, *}^{2} \times\left|v_{-}\right|_{5}^{2},
\end{aligned}
$$

where we have used Lemma 3.2 and Corollary 3.7 in the last line (notice that $\ell_{j} \leq 2$ for $j \geq 2$ ). Hence we obtain

$$
\left|\tilde{\mathcal{M}}_{m}\left(v_{-}, v_{2}, \ldots, v_{m}\right)\right|_{0,+} \lesssim \prod_{j=2}^{m}\left|v_{j}\right|_{4, *} \times\left|v_{-}\right|_{5} \quad \text { for } m \in\{2, \ldots, 6\} .
$$

Next we estimate the norm $\left|\hat{\mathcal{M}}_{m}^{(n)}\left(v_{-}, v_{2}, \ldots, v_{m}\right)\right|_{0,+}$ for $m \in\{2, \ldots, 6\}$ and $n=1,2$. Using Lemma 3.2 and noting that for any measurable $f:(-1,1) \rightarrow \mathbb{R}$ and $\beta \in \mathbb{R}$ we have

$$
\int_{-1}^{1}\left(1-x^{2}\right)^{\beta}\left(\int_{0}^{x} f\left(x^{\prime}\right) \mathrm{d} x^{\prime}\right)^{2} \mathrm{~d} x \lesssim \int_{-1}^{1}\left(1-x^{2}\right)^{\beta}(f(x))^{2} \mathrm{~d} x,
$$

it suffices to estimate (cf. (5.14) and (5.16))

$$
\left(1-x^{2}\right)^{4+n-\ell_{0}} \times \partial_{x}^{2} v_{-} \times \prod_{j=2}^{m} \partial_{x}^{\ell_{j}} v_{j} \quad \text { and } \quad\left(1-x^{2}\right)^{4+n-\ell_{0}} \times \partial_{x} v_{-} \times \prod_{j \notin\{1, i\}} \partial_{x}^{\ell_{j}} v_{j} \times \partial_{x}^{2} v_{i}
$$

in $L^{2}((-1,1))$, where $\sum_{j \neq 1} \ell_{j}=3+n$ and $\ell_{j} \leq 1$ for $j \geq 2$. For the first term in (5.24) we obtain

$$
\begin{aligned}
& \int_{-1}^{1}\left(1-x^{2}\right)^{8+2 n-2 \ell_{0}}\left(\partial_{x}^{2} v_{-}\right)^{2} \prod_{j=2}^{m}\left(\partial_{x}^{\ell_{j}} v_{j}\right)^{2} \mathrm{~d} x \\
& \leq \prod_{j=2}^{m}\left\|\left(1-x^{2}\right)^{\ell_{j}} \partial_{x}^{\ell_{j}} v_{j}\right\|_{C^{0}([-1,1])}^{2} \times \int_{-1}^{1}\left(1-x^{2}\right)^{2}\left(\partial_{x}^{2} v_{-}\right)^{2} \mathrm{~d} x \\
& \quad \lesssim \prod_{j=2}^{m}\left|v_{j}\right|_{4, *}^{2} \times \sum_{j=2}^{5} \int_{-1}^{1}\left(1-x^{2}\right)^{8}\left(\partial_{x}^{j} v_{-}\right)^{2} \mathrm{~d} x \lesssim \prod_{j=2}^{m}\left|v_{j}\right|_{4, *}^{2} \times\left|v_{-}\right|_{5}^{2}
\end{aligned}
$$

by Lemma 3.2 and Corollary 3.7. Similarly for the second term in (5.24)

$$
\begin{aligned}
& \int_{-1}^{1}\left(1-x^{2}\right)^{8+2 n-2 \ell_{0}}\left(\partial_{x} v_{-}\right)^{2} \prod_{j \notin\{1, i\}}\left(\partial_{x}^{\ell_{j}} v_{j}\right)^{2} \times\left(\partial_{x}^{2} v_{i}\right)^{2} \mathrm{~d} x \\
& \quad \leq \prod_{j \notin\{1, i\}}\left\|\left(1-x^{2}\right)^{\ell_{j}} \partial_{x}^{\ell_{j}} v_{j}\right\|_{C^{0}([-1,1])}^{2} \times\left\|\left(1-x^{2}\right)^{2} \partial_{x}^{2} v_{i}\right\|_{C^{0}([-1,1])}^{2} \times \int_{-1}^{1}\left(\partial_{x} v_{-}\right)^{2} \mathrm{~d} x \\
& \quad \lesssim \prod_{j=2}^{m}\left|v_{j}\right|_{4, *}^{2} \times \sum_{j=1}^{5} \int_{-1}^{1}\left(1-x^{2}\right)^{8}\left(\partial_{x}^{j} v_{-}\right)^{2} \mathrm{~d} x \lesssim \prod_{j=2}^{m}\left|v_{j}\right|_{4, *}^{2} \times\left|v_{-}\right|_{5}^{2} .
\end{aligned}
$$


In summary we obtain

$$
\left|\hat{\mathcal{M}}_{m}^{(n)}\left(v_{-}, v_{2}, \ldots, v_{m}\right)\right|_{0,+} \lesssim \prod_{j=2}^{m}\left|v_{j}\right|_{4, *} \times\left|v_{-}\right|_{5} \quad \text { for } m \in\{2, \ldots, 6\}
$$

and $n=1,2$.

As a last step, we estimate $\left|\hat{\mathcal{M}}_{m}^{(3)}\left(v_{-}, v_{2}, \ldots, v_{m}\right)\right|_{0,+}$ for $m \in\{2, \ldots, 6\}$ : First we apply Lemma 3.2 twice and use estimate (5.23), so that it suffices to estimate (cf. (5.18))

$$
\begin{aligned}
& \left(1-x^{2}\right)^{6-\ell_{0}} \times \prod_{j=m^{\prime}+1}^{m} v_{j} \times \int_{0}^{x} \partial_{x}^{2} v_{-} \prod_{j=2}^{m^{\prime}} \partial_{x} v_{j} \mathrm{~d} x_{1} \\
& \text { and }\left(1-x^{2}\right)^{6-\ell_{0}} \times \prod_{j=m^{\prime}+1}^{m} v_{j} \times \int_{0}^{x} \partial_{x} v_{-} \times \partial_{x}^{2} v_{i} \prod_{\substack{j=2 \\
j \neq i}}^{m^{\prime}} \partial_{x} v_{j} \mathrm{~d} x_{1}
\end{aligned}
$$

in $L^{2}((-1,1))$, where $m^{\prime}=7-\ell_{0}$. Using Corollary 3.7, we can factor out the term $\prod_{j=m^{\prime}+1}^{m}\left|v_{j}\right|_{4, *}$, so that - after applying Lemma 3.2 and (5.23) once more-it suffices to estimate

$$
\left(1-x^{2}\right)^{7-\ell_{0}} \times \partial_{x}^{2} v_{-} \times \prod_{j=2}^{m^{\prime}} \partial_{x} v_{j} \quad \text { and } \quad\left(1-x^{2}\right)^{7-\ell_{0}} \times \partial_{x} v_{-} \times \partial_{x}^{2} v_{i} \times \prod_{\substack{j=2 \\ j \neq i}}^{m^{\prime}} \partial_{x} v_{j}
$$

in $L^{2}((-1,1))$. This is the same situation as in $(5.24)$ with $n=3$, so that by the same reasoning (5.25) holds for $n=3$ as well.

Gathering (5.22) and (5.25) for $n=1,2,3$, we end up with (5.21).

Estimate of $\left|\mathcal{M}_{m}^{\prime}\left(v_{+}, v_{2}, \ldots, v_{m}\right)\right|_{2,+}$. We claim

$$
\left|\mathcal{M}_{m}^{\prime}\left(v_{+}, v_{2}, \ldots, v_{m}\right)\right|_{2,+} \lesssim \prod_{j=2}^{m}\left|v_{j}\right|_{4, *} \times\left|v_{+}\right|_{7} \quad \text { for } m \in\{2, \ldots, 6\} .
$$

Due to (5.21) we only need to treat cases in which at least one derivative acts on $\mathcal{M}_{m}^{\prime}\left(v_{+}, v_{2}, \ldots, v_{m}\right)$. Considering (5.13), we start by estimating terms of the form

$$
\left(1-x^{2}\right)^{\frac{\ell+3}{2}-\ell_{0}} \times \partial_{x}^{\ell_{1}} v_{+} \times \prod_{j=2}^{m} \partial_{x}^{\ell_{j}} v_{j} \quad \text { with } \sum_{j=0}^{m} \ell_{j}=: \ell \in\{6,7\} \text { and } \ell_{1} \geq 2 \text { if } \ell=7
$$

in $L^{2}((-1,1))$. Several cases have to be considered.

Assuming $\ell_{j} \leq 3$ for $j \geq 2$ and $\ell_{1} \geq 2$, we may estimate (5.27) as follows:

$$
\begin{aligned}
& \int_{-1}^{1}\left(1-x^{2}\right)^{\ell+3-2 \ell_{0}}\left(\partial_{x}^{\ell_{1}} v_{+}\right)^{2} \prod_{j=2}^{m}\left(\partial_{x}^{\ell_{j}} v_{j}\right)^{2} \mathrm{~d} x \\
& \leq \prod_{j=2}^{m}\left\|\left(1-x^{2}\right)^{\ell_{j}} \partial_{x}^{\ell_{j}} v_{j}\right\|_{C^{0}([-1,1])}^{2} \times \int_{-1}^{1}\left(1-x^{2}\right)^{3+2 \ell_{1}-\ell}\left(\partial_{x}^{\ell_{1}} v_{+}\right)^{2} \mathrm{~d} x \\
& \quad \lesssim \prod_{j=2}^{m}\left|v_{j}\right|_{4, *}^{2} \times \sum_{j=\ell_{1}}^{\ell} \int_{-1}^{1}\left(1-x^{2}\right)^{\ell+3}\left(\partial_{x}^{j} v_{+}\right)^{2} \mathrm{~d} x \leq \prod_{j=2}^{m}\left|v_{j}\right|_{4, *}^{2} \times\left|v_{+}\right|_{7}^{2},
\end{aligned}
$$

where we have used Lemma 3.2 and Corollary 3.7. 
If $\ell_{1} \geq 2$ and $\ell_{2}=4$, necessarily $\ell_{j} \leq 1$ for $j \geq 2$ and we similarly obtain for

$$
\begin{aligned}
\int_{-1}^{1} & \left(1-x^{2}\right)^{\ell+3-2 \ell_{0}}\left(\partial_{x}^{\ell_{1}} v_{+}\right)^{2} \prod_{j=2}^{m}\left(\partial_{x}^{\ell_{j}} v_{j}\right)^{2} \mathrm{~d} x \\
\leq & \prod_{j=3}^{m}\left\|\left(1-x^{2}\right)^{\ell_{j}} \partial_{x}^{\ell_{j}} v_{j}\right\|_{C^{0}([-1,1])}^{2} \times\left\|\left(1-x^{2}\right)^{\ell_{1}-\frac{3}{2}} \partial_{x}^{\ell_{1}} v_{+}\right\|_{C^{0}([-1,1])}^{2} \\
& \times \int_{-1}^{1}\left(1-x^{2}\right)^{14-\ell}\left(\partial_{x}^{4} v_{2}\right)^{2} \mathrm{~d} x \\
& \lesssim \prod_{j=3}^{m}\left|v_{j}\right|_{4, *}^{2} \times\left|v_{+}\right|_{7}^{2} \times\left|v_{2}\right|_{4}^{2} \leq \prod_{j=2}^{m}\left|v_{j}\right|_{4, *}^{2} \times\left|v_{+}\right|_{7}^{2}
\end{aligned}
$$

where we have used Lemma 3.4, Lemma 3.6, and Corollary 3.7.

For $\ell=6$ and $\ell_{1}=1$, we only need to consider $\ell_{j} \leq 1$ for $j \geq 2$ in (5.27) by our choice of $\hat{\mathcal{M}}_{m}^{(1)}$ (cf. (5.14)). Furthermore, by reordering we can assume without loss of generality $\ell_{2}=1$ and estimate (5.27) as follows:

$$
\begin{aligned}
& \int_{-1}^{1}\left(1-x^{2}\right)^{9-2 \ell_{0}}\left(\partial_{x} v_{+}\right)^{2} \prod_{j=2}^{m}\left(\partial_{x}^{\ell_{j}} v_{j}\right)^{2} \mathrm{~d} x \\
& \quad \leq \prod_{j=3}^{m}\left\|\left(1-x^{2}\right)^{\ell_{j}} \partial_{x}^{\ell_{j}} v_{j}\right\|_{C^{0}([-1,1])}^{2} \times\left\|\partial_{x} v_{+}\right\|_{C^{0}([-1,1])}^{2} \times \int_{-1}^{1}\left(1-x^{2}\right)\left(\partial_{x} v_{2}\right)^{2} \mathrm{~d} x \\
& \quad \lesssim \prod_{j=3}^{m}\left|v_{j}\right|_{4, *}^{2} \times\left|v_{+}\right|_{6, *}^{2} \times \sum_{j=1}^{4} \int_{-1}^{1}\left(1-x^{2}\right)^{7}\left(\partial_{x}^{j} v_{2}\right)^{2} \mathrm{~d} x \lesssim \prod_{j=2}^{m}\left|v_{j}\right|_{4, *}^{2} \times\left|v_{+}\right|_{7}^{2},
\end{aligned}
$$

where we have used Lemma 3.2, Lemma 3.6, and Corollary 3.7.

Considering $\partial_{x} \hat{\mathcal{M}}_{m}^{(2)}\left(v_{+}, v_{2}, \ldots, v_{m}\right)$, where $m \in\{2, \ldots, 6\}$, we need to estimate terms of the form (cf. (5.16))

$$
\left(1-x^{2}\right)^{\frac{5}{2}} \int_{0}^{x} \partial_{x}^{\ell_{0}}\left(1-x_{1}^{2}\right)^{2} \partial_{x}^{2} v_{+} \prod_{j=2}^{m} \partial_{x}^{\ell_{j}} v_{j} \mathrm{~d} x_{1}
$$

and

$$
\left(1-x^{2}\right)^{\frac{5}{2}} \int_{0}^{x} \partial_{x}^{\ell_{0}}\left(1-x_{1}^{2}\right)^{2} \partial_{x} v_{+} \times \partial_{x}^{2} v_{i} \prod_{j \notin\{1, i\}} \partial_{x}^{\ell_{j}} v_{j} \mathrm{~d} x_{1}
$$

in $L^{2}((-1,1))$, where $\sum_{j \neq 1} \ell_{j}=5$ and $\ell_{j} \leq 1$ for $j \geq 2$. Using Lemma 3.2 in combination with (5.23), it suffices to estimate

$$
\left(1-x^{2}\right)^{\frac{11-2 \ell_{0}}{2}} \times \partial_{x}^{2} v_{+} \times \prod_{j=2}^{m} \partial_{x}^{\ell_{j}} v_{j} \quad \text { and } \quad\left(1-x^{2}\right)^{\frac{11-2 \ell_{0}}{2}} \times \partial_{x} v_{+} \times \partial_{x}^{2} v_{i} \times \prod_{j \notin\{1, i\}} \partial_{x}^{\ell_{j}} v_{j}
$$


in $L^{2}((-1,1))$, where $\sum_{j \neq 1} \ell_{j}=5$ and $\ell_{j} \leq 1$ for $j \geq 2$. For the first term in (5.28) we obtain (we take $n=2$ )

$$
\begin{aligned}
& \int_{-1}^{1}\left(1-x^{2}\right)^{7+2 n-2 \ell_{0}}\left(\partial_{x}^{2} v_{+}\right)^{2} \prod_{j=2}^{m}\left(\partial_{x}^{\ell_{j}} v_{j}\right)^{2} \mathrm{~d} x \\
& \quad \leq \prod_{j=2}^{m}\left\|\left(1-x^{2}\right)^{\ell_{j}} \partial_{x}^{\ell_{j}} v_{j}\right\|_{C^{0}([-1,1])}^{2} \times \int_{-1}^{1}\left(1-x^{2}\right)\left(\partial_{x}^{2} v_{+}\right)^{2} \mathrm{~d} x \\
& \quad \lesssim \prod_{j=2}^{m}\left|v_{j}\right|_{4, *}^{2} \times\left|v_{+}\right|_{6}^{2} \lesssim \prod_{j=2}^{m}\left|v_{j}\right|_{4, *}^{2} \times\left|v_{+}\right|_{7}^{2}
\end{aligned}
$$

by Lemma 3.2 and Corollary 3.7. For the second term in (5.28) we may estimate as follows:

$$
\begin{aligned}
& \int_{-1}^{1}\left(1-x^{2}\right)^{7+2 n-2 \ell_{0}}\left(\partial_{x} v_{+}\right)^{2} \prod_{j \notin\{1, i\}}\left(\partial_{x}^{\ell_{j}} v_{j}\right)^{2} \times\left(\partial_{x}^{2} v_{i}\right)^{2} \mathrm{~d} x \\
& \quad \leq \prod_{j \notin\{1, i\}}\left\|\left(1-x^{2}\right)^{\ell_{j}} \partial_{x}^{\ell_{j}} v_{j}\right\|_{C^{0}([-1,1])}^{2} \times\left\|\partial_{x} v_{+}\right\|_{C^{0}([-1,1])}^{2} \times \int_{-1}^{1}\left(1-x^{2}\right)^{3}\left(\partial_{x}^{2} v_{i}\right)^{2} \mathrm{~d} x \\
& \quad \lesssim \prod_{j \notin\{1, i\}}\left|v_{j}\right|_{4, *}^{2} \times\left|v_{+}\right|_{6, *}^{2} \times\left|v_{i}\right|_{4}^{2} \lesssim \prod_{j=2}^{m}\left|v_{j}\right|_{4, *}^{2} \times\left|v_{+}\right|_{7}^{2},
\end{aligned}
$$

where we have used Lemma 3.2, Lemma 3.6, and Corollary 3.7.

For estimating $\partial_{x} \hat{\mathcal{M}}_{m}^{(3)}\left(v_{+}, v_{2}, \ldots, v_{m}\right)$, where $m \in\{2, \ldots, 6\}$, we apply Lemma 3.2 once and use inequality (5.23), so that it suffices to estimate (cf. (5.18))

$$
\left(1-x^{2}\right)^{\frac{11-2 \ell_{0}}{2}} \prod_{j=m^{\prime}+1}^{m} v_{j} \int_{0}^{x} \partial_{x}^{2} v_{+} \prod_{j=2}^{m^{\prime}} \partial_{x} v_{j} \mathrm{~d} x_{1}
$$

and

$$
\left(1-x^{2}\right)^{\frac{11-2 \ell_{0}}{2}} \prod_{j=m^{\prime}+1}^{m} v_{j} \int_{0}^{x} \partial_{x} v_{+} \times \partial_{x}^{2} v_{i} \prod_{\substack{j=2 \\ j \neq i}}^{m^{\prime}} \partial_{x} v_{j} \mathrm{~d} x_{1}
$$

in $L^{2}((-1,1))$, where $m^{\prime}=7-\ell_{0}$. By using Corollary 3.7, we can factor out $\prod_{j=m^{\prime}+1}^{m}\left|v_{j}\right|_{4, *}$ in both cases so that - after applying Lemma 3.2 and using (5.23) - it suffices to estimate

$$
\left(1-x^{2}\right)^{\frac{13-2 \ell_{0}}{2}} \partial_{x}^{2} v_{+} \times \prod_{j=2}^{m^{\prime}} \partial_{x} v_{j} \quad \text { and } \quad\left(1-x^{2}\right)^{\frac{13-2 \ell_{0}}{2}} \partial_{x} v_{+} \times \partial_{x}^{2} v_{i} \times \prod_{\substack{j=2 \\ j \neq i}}^{m^{\prime}} \partial_{x} v_{j}
$$

in $L^{2}((-1,1))$. These terms have been estimated after (5.28) when setting $n=3$ and replacing $m$ by $m^{\prime}$ and are estimated by $\prod_{j=2}^{m^{\prime}}\left|v_{j}\right|_{4, *}^{2} \times\left|v_{+}\right|_{7}^{2}$ as desired. 
As a last step, we treat the case in which $\ell_{1}=1$ and $\ell=7$ in the term in (5.27). Due to the definition of $\hat{\mathcal{M}}_{m}$ (cf. (5.14), (5.16), (5.18)), we may estimate the sum

$$
\begin{aligned}
& \left(1-x^{2}\right)^{5-\ell_{0}} \partial_{x} v_{+} \prod_{j=2}^{m^{\prime}} \partial_{x} v_{j} \prod_{j=m^{\prime}+1}^{m} v_{j} \\
& +m^{\prime}\left(1-x^{2}\right)^{5-\ell_{0}} \prod_{j=m^{\prime}+1}^{m} v_{j} \int_{0}^{x} \partial_{x}^{2} v_{+} \prod_{j=2}^{m^{\prime}} \partial_{x} v_{j} \mathrm{~d} x_{1} \\
& -\sum_{i=1}^{m^{\prime}}\left(1-x^{2}\right)^{5-\ell_{0}} \prod_{j=m^{\prime}+1}^{m} v_{j} \int_{0}^{x} \partial_{x} v_{+} \times \partial_{x}^{2} v_{i} \prod_{\substack{j=2 \\
j \neq i}}^{m^{\prime}} \partial_{x} v_{j} \mathrm{~d} x_{1}
\end{aligned}
$$

in $L^{2}((-1,1))$, where $m^{\prime}=7-\ell_{0}$. By Corollary 3.7 , we first factor out the product $\prod_{j=m^{\prime}+1}^{m}\left|v_{j}\right|_{4, *}$. Using Lemma 3.2 once, it then suffices to estimate

$$
\left(1-x^{2}\right)^{6-\ell_{0}} \times \partial_{x}^{2} v_{+} \times \prod_{j=2}^{m^{\prime}} \partial_{x} v_{j}
$$

as well as (using inequality (5.23))

$$
\begin{aligned}
& \left(1-x^{2}\right)^{6-\ell_{0}} \times \partial_{x} v_{+} \times \prod_{j=2}^{m^{\prime}} \partial_{x} v_{j}, \\
& \left(1-x^{2}\right)^{6-\ell_{0}} \times \partial_{x}^{2} v_{+} \times \prod_{j=2}^{m^{\prime}} \partial_{x} v_{j}, \\
& \left(1-x^{2}\right)^{\frac{13-2 \ell_{0}}{2}} \times \partial_{x} v_{+} \times \partial_{x}^{2} v_{i} \times \prod_{\substack{j=2 \\
j \neq i}}^{m^{\prime}} \partial_{x} v_{j}
\end{aligned}
$$

in $L^{2}((-1,1))$. For $(5.30)$ we can use the $L^{2}$-bound on $\partial_{x}^{2} v_{+}$and obtain

$$
\begin{aligned}
& \int_{-1}^{1}\left(1-x^{2}\right)^{12-2 \ell_{0}}\left(\partial_{x}^{2} v_{+}\right)^{2} \prod_{j=2}^{m^{\prime}}\left(\partial_{x} v_{j}\right)^{2} \mathrm{~d} x \\
& \quad \lesssim \prod_{j=2}^{m^{\prime}}\left\|\left(1-x^{2}\right) \partial_{x} v_{j}\right\|_{C^{0}([-1,1])}^{2} \times \int_{-1}^{1}\left(\partial_{x}^{2} v_{+}\right)^{2} \mathrm{~d} x \\
& \quad \lesssim \prod_{j=2}^{m^{\prime}}\left|v_{j}\right|_{4, *}^{2} \times \sum_{j=2}^{7} \int_{-1}^{1}\left(1-x^{2}\right)^{10}\left(\partial_{x}^{j} v_{+}\right)^{2} \mathrm{~d} x \lesssim \prod_{j=2}^{m^{\prime}}\left|v_{j}\right|_{4, *}^{2} \times\left|v_{+}\right|_{7}^{2}
\end{aligned}
$$

where we have used Lemma 3.2 and Corollary 3.7. The first term in (5.31) can be treated in the same way and we obtain

$$
\begin{aligned}
& \int_{-1}^{1}\left(1-x^{2}\right)^{12-2 \ell_{0}}\left(\partial_{x} v_{+}\right)^{2} \prod_{j=2}^{m^{\prime}}\left(\partial_{x} v_{j}\right)^{2} \mathrm{~d} x \\
& \quad \lesssim \prod_{j=2}^{m}\left\|\left(1-x^{2}\right) \partial_{x} v_{j}\right\|_{C^{0}([-1,1])}^{2} \times \int_{-1}^{1}\left(\partial_{x} v_{+}\right)^{2} \mathrm{~d} x \lesssim \prod_{j=2}^{m}\left|v_{j}\right|_{4, *}^{2} \times\left|v_{+}\right|_{5}^{2} .
\end{aligned}
$$


The second term in (5.31) is the same as in (5.30) and the last term already appeared in (5.29) and was treated after (5.28).

Gathering all previous estimates, we obtain (5.26).

Conclusion. We use estimates (5.21) and (5.26) in (5.20) and obtain (using Lemma 3.8)

$$
\begin{gathered}
\int_{0}^{\infty} \inf _{v_{1}=v_{-}+v_{+}}\left(\int _ { I } e ^ { 2 \mu \tau } \left(\sigma^{-1}\left|\mathcal{M}_{m}^{\prime}\left(v_{-}, v_{2}, \ldots, v_{m}\right)\right|_{0,+}^{2}\right.\right. \\
\left.\left.+\sigma\left|\mathcal{M}_{m}^{\prime}\left(v_{+}, v_{2}, \ldots, v_{m}\right)\right|_{2,+}^{2}\right) \mathrm{~d} \tau\right)^{\frac{1}{2}} \frac{\mathrm{d} \sigma}{\sigma} \\
\lesssim \int_{0}^{\infty} \inf _{v_{1}=v_{-}+v_{+}}\left(\int _ { I } e ^ { 2 \mu \tau } \left(\sigma^{-1} \prod_{j=2}^{m}\left|v_{j}\right|_{4, *}^{2} \times\left|v_{-}\right|_{5}^{2}\right.\right. \\
\left.\left.+\sigma \prod_{j=2}^{m}\left|v_{j}\right|_{4, *}^{2} \times\left|v_{+}\right|_{7}^{2}\right) \mathrm{~d} \tau\right)^{\frac{1}{2}} \frac{\mathrm{d} \sigma}{\sigma} \\
\lesssim \prod_{j=2}^{m}\left\|v_{j}\right\|_{C_{\mu}^{0}\left(\bar{I} ; \mathcal{H}^{4}\right)^{*}}^{2} \times\left\|v_{1}\right\|_{L_{\mu}^{2}\left(I ; \mathcal{H}^{6}\right)^{*}}^{2} \lesssim \prod_{j=1}^{m}\left\|v_{j}\right\|_{I}^{2},
\end{gathered}
$$

where $m \in\{2, \ldots, 6\}, v_{j} \in\left\{u_{1}, u_{2}, u_{1}-u_{2}\right\}$, and $v_{j}=u_{1}-u_{2}$ for exactly one $j \in\{1, \ldots, m\}$. Since the sum of terms of the form (5.20) bounds the interpolation seminorm in (5.6), we obtain

$$
\begin{aligned}
& \int_{0}^{\infty} \inf _{\mathcal{N}\left(u_{1}\right)-\mathcal{N}\left(u_{2}\right)=N_{-}+N_{+}}\left(\int_{0}^{\infty} e^{2 \mu \tau}\left(\sigma^{-1}\left[N_{-}\right]_{1}^{2}+\sigma\left[N_{+}\right]_{1,3}^{2}\right) \mathrm{d} \tau\right)^{\frac{1}{2}} \frac{\mathrm{d} \sigma}{\sigma} \\
& \quad \lesssim \max _{m=1,5}\left(\left\|u_{1}\right\|_{I}^{m}+\left\|u_{2}\right\|_{I}^{m}\right)\|\| u_{1}-u_{2} \|_{I} .
\end{aligned}
$$

The combination of (5.7) and (5.32) yields (5.5b) and concludes the proof.

6. Existence and uniqueness. We are now ready to prove Theorem 2.1, which is a consequence of the more general statement as follows.

Proposition 6.1. Suppose $\mu<120$. Then there exists a constant $\delta>0$ independent of $\mu$ such that for every initial value $u_{0} \in \mathcal{H}_{*}^{4}$ with $\left\|u_{0}\right\|_{0} \leq \delta$, there exists a unique solution

$$
u \in H_{\mu}^{1}\left((0, \infty) ; \mathcal{H}^{2}\right)^{*} \cap C_{\mu}^{0}\left([0, \infty) ; \mathcal{H}^{4}\right)^{*} \cap L_{\mu}^{2}\left((0, \infty) ; \mathcal{H}^{6}\right)^{*}
$$

of problem (1.20). This solution obeys the a priori estimate

$$
\|u \mid\| \lesssim_{\mu}\left\|u_{0}\right\|_{0} \text {. }
$$

Proof. It suffices to prove Proposition 6.1 with $\delta$ depending on $\mu$ : Taking $\mu:=60$ and noticing that $\left\|\left.\left|u(\tau) \|_{0}=\right| u(\tau)\right|_{4, *} \rightarrow 0\right.$ as $\tau \rightarrow \infty$ by (6.1) (cf. (4.1a)), we can solve (1.20) starting from time $\tau_{0} \gg_{\mu} 1$ (where now $\mu<120$ is arbitrary), so that, by existence and uniqueness, regularity and the a priori estimate (6.1) hold for all $\mu<120$ and $\delta>0$ fixed.

We apply the linear solution operator $\mathcal{T}$, constructed in Proposition 4.1 and obeying estimates (4.2), to the nonlinear problem (1.20) and obtain the fixed point equation

$$
u=\mathcal{T}\left[u_{0}, \mathcal{N}(u)\right]=: \mathcal{S}(u),
$$

where we keep $u_{0} \in \mathcal{H}_{*}^{4}$ fixed. 
Existence. Our aim is to prove that $\mathcal{S}$ is a self-map in the space $\{u:\|u\| \leq \varepsilon\}$, provided $\left\|u_{0}\right\|_{0} \leq \delta$ and $0<\delta \ll \varepsilon \ll_{\mu} 1$. For this purpose we notice that by the linear estimate (4.2) of Proposition 4.1 and the nonlinear estimate (5.5a) of Proposition 5.2, we have for $\varepsilon \leq 1$

$$
\|\mathcal{S}(u)\| \stackrel{(6.2)}{=}\left\|\mathcal{T}\left[u_{0}, \mathcal{N}(u)\right]\right\| \stackrel{(4.2)}{\lesssim}\left\|u_{0}\right\|_{0}+\|\mathcal{N}(u)\|_{1} \stackrel{(5.5 a)}{\lesssim} \delta+\max _{m=2, \cdots, 6}\|u\|^{m} \leq \delta+\varepsilon^{2} .
$$

Hence $\mathcal{S}$ maps $\{u:|\|u\|| \leq \varepsilon\}$ into itself if $0<\delta \ll \varepsilon \ll_{\mu} 1$. For the contraction property we use the linearity of $\mathcal{T}$ and similarly obtain

$$
\begin{aligned}
\left\|\mathcal{S}\left(u_{1}\right)-\mathcal{S}\left(u_{2}\right)\right\| & \stackrel{(6.2)}{=}\left\|\mathcal{T}\left[0, \mathcal{N}\left(u_{1}\right)-\mathcal{N}\left(u_{2}\right)\right]\right\| \stackrel{(4.2)}{\lesssim \mu}\left\|\mathcal{N}\left(u_{1}\right)-\mathcal{N}\left(u_{2}\right)\right\|_{1} \\
& \stackrel{(5.5 \mathrm{~b})}{\lesssim} \max _{m=1,5}\left(\left\|u_{1}\right\|^{m}+\left.\left\|u_{2}\right\|\right|^{m}\right)\left\|u_{1}-u_{2}\right\| \lesssim \varepsilon\left\|u_{1}-u_{2}\right\| .
\end{aligned}
$$

This shows that $\mathcal{S}$ is a contraction provided $\varepsilon \ll_{\mu} 1$. The contraction mapping theorem yields a unique fixed point which is a solution to the nonlinear Cauchy problem (1.20).

Uniqueness. Suppose that there are two solutions $u_{1}$ and $u_{2}$ for the same initial value $u_{0} \in \mathcal{H}_{*}^{4}$. Then for any interval $I=\left(0, \tau_{1}\right) \subseteq(0, \infty)$ we have

$$
\begin{aligned}
& \left\|u_{1}-u_{2}\right\|_{I} \stackrel{(6.2)}{=} \quad\left\|\mathcal{T}\left[0, \mathcal{N}\left(u_{1}\right)-\mathcal{N}\left(u_{2}\right)\right]\right\|_{I} \\
& \stackrel{(4.2),(5.5 \mathrm{~b})}{\sum_{\mu}} \max _{m=1,5}\left(\left\|u_{1}\right\|_{I}^{m}+\left\|u_{2}\right\|_{I}^{m}\right)\left\|u_{1}-u_{2}\right\|_{I} .
\end{aligned}
$$

Now, from the definition of the norms and since we have $\left.u_{j}\right|_{t=0}=u_{0}(j=1,2)$, we have $\left\|u_{j}\right\|_{I} \rightarrow\left\|u_{0}\right\|_{0} \leq \delta$ as $\tau_{1} \searrow 0$ (since the $L^{2}$-parts in the norm $\|\cdot \cdot\|_{I}$ converge to 0 by dominated convergence). Hence for sufficiently small $\tau_{1}>0$ and $\delta \ll_{\mu} 1$, we obtain from (6.4) that $\left\|u_{1}-u_{2}\right\|_{I} \leq 0$ and therefore $u_{1}(\tau, x)=u_{2}(\tau, x)$ everywhere in $(\tau, x) \in\left(0, \tau_{1}\right) \times[-1,1]$. A contradiction argument then yields global uniqueness.

7. Regularity. In this section we prove that the unique solution $u$ to (1.20) for initial data $u_{0} \in \mathcal{H}_{*}^{4}$ with $\left\|\left.u_{0}\left|\|_{0}=\right| u_{0}\right|_{4, *} \ll 1\right.$ (cf. Theorem 2.1) not only obeys

$$
u \in H^{1}\left((0, \infty) ; \mathcal{H}^{2}\right)^{*} \cap C^{0}\left([0, \infty) ; \mathcal{H}^{4}\right)^{*} \cap L^{2}\left((0, \infty) ; \mathcal{H}^{6}\right)^{*}
$$

but gains regularity for sufficiently large times. We prove this assertion iteratively and concentrate on the estimates.

We use the following crucial estimate for the nonlinearity.

Lemma 7.1. Suppose $k \geq 0$. Then

$$
|\mathcal{N}(u)|_{k} \lesssim_{k} \max _{m=1,5}|u|_{4, *}^{m} \times|u|_{k+4} \quad \text { for all } u \in \mathcal{H}^{k+4}
$$

Proof. Again, due to (1.21), derivatives $\partial_{x}^{k} \mathcal{N}(u)$ of the nonlinearity are linear combinations of

$$
\partial_{x}^{\ell_{0}}\left(1-x^{2}\right)^{2} \times \prod_{j=1}^{m} \partial_{x}^{\ell_{j}} u \quad \text { with } m \in\{2, \ldots, 6\} \text { and } \sum_{j=0}^{m} \ell_{j}=: \ell=k+4 .
$$


Hence, for establishing (7.1), it suffices to estimate terms of the form

$$
\left(1-x^{2}\right)^{\frac{\ell-1}{2}+2-\ell_{0}} \times \prod_{j=1}^{m} \partial_{x}^{\ell_{j}} u \quad \text { with } m \in\{2, \ldots, 6\} \text { and } \sum_{j=0}^{m} \ell_{j}=: \ell \leq k+4
$$

in $L^{2}((-1,1))$. Applying Lemma $3.2 \ell_{0}$-times to $(7.2)$, it suffices to estimate the following nonlinear term:

$$
\left|\prod_{j=1}^{m}\left(1-x^{2}\right)^{\frac{\ell_{j}}{2}} \partial_{x}^{\ell_{j}} u\right|_{0}^{2}=\int_{-1}^{1}\left(1-x^{2}\right)^{3} \prod_{j=1}^{m}\left(1-x^{2}\right)^{\ell_{j}}\left(\partial_{x}^{\ell_{j}} u\right)^{2} \mathrm{~d} x
$$

where $m \in\{2, \ldots, 6\}, \ell_{i} \geq \ell_{j}$ for $i \leq j$, and $\ell:=\sum_{j=1}^{m} \ell_{j} \leq k+4$. Then we distinguish between two cases.

Case I. We assume $\ell_{1} \geq 4$. Then we can estimate (7.3) as

$$
\begin{aligned}
\left|\prod_{j=1}^{m}\left(1-x^{2}\right)^{\frac{\ell_{j}}{2}} \partial_{x}^{\ell_{j}} u\right|_{0} & \leq \prod_{j=2}^{m} \|\left.\left(1-x^{2}\right)^{\frac{\ell_{j}}{2}} \partial_{x}^{\ell_{j}} u\right|_{C^{0}([-1,1])} \times|u|_{\ell_{1}} \\
& \lesssim_{k} \prod_{j=2}^{m} \max \left\{|u|_{4+\ell_{j}},|u|_{4, *}\right\} \times|u|_{\ell_{1}},
\end{aligned}
$$

where we have used Lemma 3.4 and Corollary 3.7 in the second estimate. Since the indices on the right-hand side sum to $4(m-1)+\ell, 4 \leq \ell_{1} \leq \ell$, and $4 \leq 4+\ell_{j} \leq \ell$ for $j=2, \ldots, m$, we can apply the interpolation estimate (3.8) of Lemma 3.6 and so obtain

$$
\left|\prod_{j=1}^{m}\left(1-x^{2}\right)^{\frac{\ell_{j}}{2}} \partial_{x}^{\ell_{j}} u\right|_{0} \lesssim_{k}|u|_{4, *}^{m-1} \times|u|_{\ell}
$$

The right-hand side of (7.4) is estimated by the right-hand side of (7.1).

Case II. Suppose $\ell_{1} \leq 3$. Pick $m^{\prime}$ maximal such that $\ell^{\prime}:=\sum_{j=2}^{m^{\prime}} \ell_{j} \leq \ell_{1}+2$. Then

$$
\begin{aligned}
& \int_{-1}^{1}\left(1-x^{2}\right)^{\ell+3} \prod_{j=1}^{m}\left(\partial_{x}^{\ell_{j}} u\right)^{2} \mathrm{~d} x \\
& \leq \prod_{j=m^{\prime}+1}^{m}\left\|\left(1-x^{2}\right)^{\frac{\ell_{j}}{2}} \partial_{x}^{\ell \ell_{j}} u\right\|_{C^{0}([-1,1])}^{2} \times \prod_{j=2}^{m^{\prime}}\left\|\left(1-x^{2}\right)^{\ell_{j}} \partial_{x}^{\ell_{j}} u\right\|_{C^{0}([-1,1])}^{2} \\
& \quad \times \int_{-1}^{1}\left(1-x^{2}\right)^{3+\ell_{1}-\ell^{\prime}}\left(\partial_{x}^{\ell_{1}} u\right)^{2} \mathrm{~d} x \\
& \lesssim_{k} \prod_{j=m^{\prime}+1}^{m} \max \left\{|u|_{4+\ell_{j}},|u|_{4, *}\right\} \times|u|_{4, *}^{2\left(m^{\prime}-1\right)} \times \sum_{j=\ell_{1}}^{\ell_{1}+\ell^{\prime}} \int_{-1}^{1}\left(1-x^{2}\right)^{3+\ell_{1}+\ell^{\prime}}\left(\partial_{x}^{j} u\right)^{2} \mathrm{~d} x \\
& \leq \prod_{j=m^{\prime}+1}^{m} \max \left\{|u|_{4+\ell_{j}},|u|_{4, *}\right\} \times|u|_{4, *}^{2\left(m^{\prime}-1\right)} \times|u|_{\ell_{1}+\ell^{\prime}}^{2} .
\end{aligned}
$$


Here we have used Lemma 3.2, Lemma 3.4, and Corollary 3.7. Then again the indices of the norms sum to $4(m-1)+\ell, \min \{4, \ell\} \leq \ell_{1}+\ell^{\prime} \leq \ell$, and $4 \leq 4+\ell_{j} \leq \max \{4, \ell\}$ for $j \geq m^{\prime}+1$. Hence by interpolation (Lemma 3.6), we obtain (7.4) again.

Proof of Theorem 2.2. Again, we concentrate on deriving estimates heuristically. For qualitative arguments concerning smoothness we refer to [17, section 8].

By similar arguments as in the proof of Proposition 6.1, it suffices to prove Theorem 2.2 for $\tau_{k}$ depending on $\mu$. By testing (1.20a) appropriately, we deduce

$$
\frac{\mathrm{d}}{\mathrm{d} \tau} e^{2 \mu \tau}(u,(\mathcal{L}-\mu) u)_{k}+e^{2 \mu \tau}|(\mathcal{L}-\mu) u|_{k}^{2} \lesssim_{\mu} e^{2 \mu \tau}|\mathcal{N}(u)|_{k}^{2}, \quad \text { where } \mu<120 .
$$

We multiply (7.6) with $\left(\tau-\tau_{k}\right)^{\frac{k-2}{2}}$ where $k \geq 4$ and integrate the resulting expression in time $\tau$ from $\tau_{k} \geq 0$, so that (employing Corollary 4.3)

$$
\sup _{\tau \geq \tau_{k}}\left(\tau-\tau_{k}\right)^{\frac{k-2}{2}} e^{2 \mu \tau}|u|_{k+2}^{2}+\int_{\tau_{k}}^{\infty}\left(\tau-\tau_{k}\right)^{\frac{k-2}{2}} e^{2 \mu \tau}|u|_{k+4}^{2} \mathrm{~d} \tau
$$

$$
\lesssim_{\mu, k} \int_{\tau_{k}}^{\infty}\left(\tau-\tau_{k}\right)^{\frac{k-2}{2}} e^{2 \mu \tau}|\mathcal{N}(u)|_{k}^{2} \mathrm{~d} \tau+(k-2) \int_{\tau_{k}}^{\infty}\left(\tau-\tau_{k}\right)^{\frac{k-4}{2}} e^{2 \mu \tau}|u|_{k+2}^{2} \mathrm{~d} \tau .
$$

Now we use estimate (7.1) of Lemma 7.1 in (7.7), leading to

$$
\begin{aligned}
& \sup _{\tau \geq \tau_{k}}\left(\tau-\tau_{k}\right)^{\frac{k-2}{2}} e^{2 \mu \tau}|u|_{k+2}^{2}+\int_{\tau_{k}}^{\infty}\left(\tau-\tau_{k}\right)^{\frac{k-2}{2}} e^{2 \mu \tau}|u|_{k+4}^{2} \mathrm{~d} \tau \\
& \lesssim_{\mu, k} e^{-2 \mu \tau_{k}} \max _{m=1,5} \sup _{\tau \geq \tau_{k}} e^{2 \mu \tau}|u|_{4, *}^{2 m} \times \int_{\tau_{k}}^{\infty}\left(\tau-\tau_{k}\right)^{\frac{k-2}{2}} e^{2 \mu \tau}|u|_{k+4}^{2} \mathrm{~d} \tau \\
& \quad+\int_{\tau_{k}}^{\infty}\left(\tau-\tau_{k}\right)^{\frac{k-4}{2}} e^{2 \mu \tau}|u|_{k+2}^{2} \mathrm{~d} \tau
\end{aligned}
$$

for some $\mu \in(0,120)$. We note that $e^{\mu \tau}|u|_{4, *} \stackrel{(2.1)}{\lesssim} 1$. Upon increasing the value of $\tau_{k}$, we can absorb the second line in (7.8) into the first line. Furthermore, we may use the induction assumption (already proved for $k=2$; cf. Proposition 6.1 ) for the third line in (7.8), so that

$$
\sup _{\tau \geq \tau_{k}}\left(\tau-\tau_{k}\right)^{\frac{k-2}{2}} e^{2 \mu \tau}|u|_{k+2}^{2}+\int_{\tau_{k}}^{\infty}\left(\tau-\tau_{k}\right)^{\frac{k-2}{2}} e^{2 \mu \tau}|u|_{k+4}^{2} \mathrm{~d} \tau \lesssim \mu, k \delta^{2} .
$$

By interpolation (using Lemma 3.8) and increasing $\tau_{k}$, (7.9) yields (2.2).

8. Concluding remarks. It was already suggested in [12, section. 6$]$ to investigate the asymptotics of the thin-film equation with nonzero contact angles (i.e., $\partial_{z} h=0$ at $z=Z_{ \pm}(t)$ (cf. (1.1c)) is not necessarily fulfilled). Global existence and local well-posedness for prescribed nonzero contact angle have been addressed, for instance, by Otto [30], Bertsch, Giacomelli, and Karali [7], Knüpfer and Masmoudi $[21,22]$, and Mellet [27]. Here, one expects convergence to the profile (compare to (1.4), respectively, [12, equation (6.1)])

$$
h_{\mathrm{s}, \gamma}(t, z)=(t+1)^{-\frac{1}{5}}\left(\frac{\Xi^{4}}{120}\left(1-x^{2}\right)^{2}+\frac{\gamma \Xi}{2}\left(1-x^{2}\right)\right) \quad \text { for } x \in[-1,1],
$$

where $x=\Xi^{-1}(t+1)^{-\frac{1}{5}} z, \Xi>0$ determines the mass of the droplet, and $\gamma \geq 0$ the dynamic contact angle of $h_{\mathrm{s}}$ (note that $\left.\partial_{z} h_{\mathrm{s}}\left(t, Z_{\mathrm{s}, \pm}(t)\right)=\mp \gamma(t+1)^{-\frac{2}{5}}\right) .^{2}$ Using the

\footnotetext{
${ }^{2}$ For discussions on dynamic contact angle conditions we refer to [8, 32].
} 
analogue of (1.6), i.e.,

$$
\int_{Z_{-}(t)}^{Z(t, x)} h(t, z) \mathrm{d} z:=\int_{-1}^{x}\left(\frac{\Xi^{5}}{120}\left(1-\left(x^{\prime}\right)^{2}\right)^{2}+\frac{\gamma \Xi^{2}}{2}\left(1-\left(x^{\prime}\right)^{2}\right)\right) \mathrm{d} x^{\prime}
$$

for $x \in(-1,1)$, we can carry out the transformations presented in the introduction, too. Yet, the linear operator in this case does seem to have an apparent symmetric structure as the operator $\mathcal{L}$ given in (1.19) (cf. Corollary 4.3). In particular the spectrum of the linearization is supposedly not real, so that already the linear analysis is presumably more involved.

An equally relevant question concerns stability and uniqueness issues of sourcetype self-similar solutions to the thin-film equation (1.2) with general mobility. We expect that the rather explicit characterization of the boundary regularity of sourcetype self-similar solutions in [15] and a corresponding well-posedness result in case of the half-line [14] can lead to new insights here.

\section{Appendix A. Transformations.}

Source-type self-similar solutions. Here we derive explicit expressions for the source-type self-similar solution. Inserting ansatz (1.3), that is,

$$
h_{\mathrm{s}}(t, z)=(t+1)^{-\frac{1}{5}} H_{\mathrm{s}}(\xi), \quad \text { where } \xi=(t+1)^{-\frac{1}{5}} z,
$$

into the thin-film equation (1.1a), we obtain

$$
\frac{\mathrm{d}}{\mathrm{d} \xi}\left(H_{\mathrm{s}} \frac{\mathrm{d}^{3} H_{\mathrm{s}}}{\mathrm{d} \xi^{3}}\right)=\frac{1}{5} \frac{\mathrm{d}}{\mathrm{d} \xi}\left(\xi H_{\mathrm{s}}\right)
$$

which we can integrate (using boundary conditions (1.1b)) to the result

$$
\frac{\mathrm{d}^{3} H_{\mathrm{s}}}{\mathrm{d} \xi^{3}}=\frac{\xi}{5} \quad \text { for } \xi \in\left(\xi_{-}, \xi_{+}\right) \quad \text { with } \quad H_{\mathrm{s}}=\frac{\mathrm{d} H_{\mathrm{s}}}{\mathrm{d} \xi}=0 \quad \text { at } \xi=\xi_{ \pm} .
$$

One then easily checks that for $\xi_{ \pm}= \pm \Xi$ with some $\Xi>0$, problem (A.1) has the unique solution

$$
H_{\mathrm{s}}(\xi)=\frac{1}{120}\left(\Xi^{2}-\xi^{2}\right)^{2} \quad \text { for } \xi \in\left(\xi_{-}, \xi_{+}\right),
$$

where the quantity $\Xi$ is linked to the mass $M$ of the film by

$$
M=\int_{-(t+1)^{\frac{1}{5}} \Xi}^{(t+1)^{\frac{1}{5}} \Xi} h_{\mathrm{s}}(t, z) \mathrm{d} z \stackrel{(1.3)}{=} \int_{-\Xi}^{\Xi} H_{\mathrm{S}}(\xi) \mathrm{d} \xi \stackrel{(\mathrm{A} .2)}{=} \frac{2 \Xi^{5}}{225} .
$$

See also $[5,34]$. We can further use the transformation

$$
x:=\frac{\xi}{\Xi} \stackrel{(1.3)}{=} \frac{z}{(t+1)^{\frac{1}{5}} \Xi} \stackrel{(1.4)}{=} \frac{z}{(225 M(t+1) / 2)^{\frac{1}{5}}}
$$

so that the self-similar solution attains the form (1.4). 
Linearization and nonlinear terms. In this appendix, we derive explicit expressions for the nonlinearity $\mathcal{N}(u)$ of $(1.20 \mathrm{a})$ and show how the linear operator (cf. (1.19)) can be derived. First, one may further simplify the 6-linear term

$$
N(F):=F^{2} \partial_{x}\left(F \partial_{x}\right)^{3}\left(1-x^{2}\right)^{2} F
$$

in $(1.16)$ :

$$
\begin{aligned}
N(F)= & F^{2} \partial_{x} F \partial_{x} F \partial_{x}\left(\left(1-x^{2}\right)^{2} F \partial_{x} F-4 x\left(1-x^{2}\right) F^{2}\right) \\
= & F^{2} \partial_{x} F \partial_{x}\left(1-x^{2}\right)^{2} F \partial_{x} F \partial_{x} F \\
& -12 F^{2} \partial_{x} F \partial_{x} x\left(1-x^{2}\right) F^{2} \partial_{x} F+4 F^{2} \partial_{x} F \partial_{x}\left(3 x^{2}-1\right) F^{3} \\
= & F^{2} \partial_{x}\left(1-x^{2}\right)^{2} F \partial_{x} F \partial_{x} F \partial_{x} F-4 F^{2} \partial_{x} x\left(1-x^{2}\right) F\left(3 \partial_{x} F^{2} \partial_{x} F+F \partial_{x} F \partial_{x} F\right) \\
& +24 F^{2} \partial_{x}\left(3 x^{2}-1\right) F^{3} \partial_{x} F+24 F^{2} \partial_{x} x F^{4} .
\end{aligned}
$$

Applying the last derivative and reordering the terms in the sum, we obtain

$$
N(F)=N_{4}(F)+N_{3}(F)+N_{2}(F)+N_{1}(F)+N_{0}(F)
$$

where

(A.4c) $N_{3}(F)=-4 x\left(1-x^{2}\right) F^{2}\left(3 \partial_{x} F \partial_{x} F^{2} \partial_{x} F+\partial_{x} F^{2} \partial_{x} F \partial_{x} F+F \partial_{x} F \partial_{x} F \partial_{x} F\right)$,

(A.4d) $N_{2}(F)=4\left(3 x^{2}-1\right) F^{2}\left(6 \partial_{x} F^{3} \partial_{x} F+3 F \partial_{x} F^{2} \partial_{x} F+F^{2} \partial_{x} F \partial_{x} F\right)$,

(A.4e) $N_{1}(F)=240 x F^{5} \partial_{x} F$,

(A.4f) $\quad N_{0}(F)=24 F^{6}$.

Setting $u:=F-F_{\mathrm{s}}=F-1$ as in (1.17), equations (1.16), (A.3), and (A.4) lead to the linear operator $\mathcal{L}$ (associated to (1.16)) given in (1.19) and one can prove by direct computation that it has the symmetric structure presented there. Furthermore, (1.16) can be transformed into the evolution equation (1.20a) for $u$, where the nonlinearity $\mathcal{N}(u)$ is given by

$\left(\right.$ A.5a) $\mathcal{N}(u):=\mathcal{L} u+24(1+u)-N(1+u)=\mathcal{N}_{4}(u)+\mathcal{N}_{3}(u)+\mathcal{N}_{2}(u)+\mathcal{N}_{1}(u)+\mathcal{N}_{0}(u)$

with

$$
\begin{aligned}
\mathcal{N}_{4}(u)= & \left(1-x^{2}\right)^{2}\left(\partial_{x}^{4} u-(1+u)^{2} \partial_{x}(1+u) \partial_{x}(1+u) \partial_{x}(1+u) \partial_{x} u\right), \\
\mathcal{N}_{3}(u)= & -4 x\left(1-x^{2}\right)\left(5 \partial_{x}^{3} u-3(1+u)^{2} \partial_{x}(1+u) \partial_{x}(1+u)^{2} \partial_{x} u\right. \\
& -(1+u)^{2} \partial_{x}(1+u)^{2} \partial_{x}(1+u) \partial_{x} u \\
& \left.-(1+u)^{3} \partial_{x}(1+u) \partial_{x}(1+u) \partial_{x} u\right) \\
\mathcal{N}_{2}(u)= & 4\left(3 x^{2}-1\right)\left(10 \partial_{x}^{2} u-6(1+u)^{2} \partial_{x}(1+u)^{3} \partial_{x} u\right. \\
& \left.-3(1+u)^{3} \partial_{x}(1+u)^{2} \partial_{x} u-(1+u)^{4} \partial_{x}(1+u) \partial_{x} u\right) \\
\mathcal{N}_{1}(u)= & 240 x\left(\partial_{x} u-(1+u)^{5} \partial_{x} u\right)
\end{aligned}
$$

and

$$
\mathcal{N}_{0}(u)=24\left(1+6 u-(1+u)^{6}\right) .
$$

One may also verify that (A.5) are compatible with the structure (1.21) for $\mathcal{N}(u)$. 
Appendix B. Weighted Sobolev spaces and their interpolation spaces. In this appendix we provide the remaining proofs of section 3 .

Proof of Lemma 3.2. We first apply the splitting

$$
\int_{-1}^{1}\left(1-x^{2}\right)^{\beta} v^{2} \mathrm{~d} x=\int_{-1}^{0}\left(1-x^{2}\right)^{\beta} v^{2} \mathrm{~d} x+\int_{0}^{1}\left(1-x^{2}\right)^{\beta} v^{2} \mathrm{~d} x .
$$

Since the terms on the right-hand side of (B.1) are symmetric, we concentrate on estimating the first term. Take a smooth cut off $\eta(x)$ with $\eta(x) \equiv 1$ for $x \leq 0$ and $\eta(x) \equiv 0$ for $x \geq \frac{1}{2}$ and set $w(y):=v(x) \eta(x)$ with $x+1=: y$. Then we can estimate

$$
\int_{-1}^{0}\left(1-x^{2}\right)^{\beta} v^{2} \mathrm{~d} x \lesssim \beta \int_{-1}^{0}(1+x)^{\beta} v^{2} \mathrm{~d} x=\int_{0}^{1} y^{\beta} w^{2} \mathrm{~d} y \leq \int_{0}^{\infty} y^{\beta} w^{2} \mathrm{~d} y .
$$

For the last term we apply Hardy's inequality (cf. Lemma 3.1) and so obtain

$$
\int_{-1}^{0}\left(1-x^{2}\right)^{\beta} v^{2} \mathrm{~d} x \lesssim_{\beta} \int_{0}^{\infty} y^{\beta+2}\left(\partial_{y} w\right)^{2} \mathrm{~d} y \quad \text { for } \beta>-1
$$

Since

$$
\left(\partial_{y} w\right)^{2}=\left(v \partial_{x} \eta+\eta \partial_{x} v\right)^{2} \leq 2\left(\left(v \partial_{x} \eta\right)^{2}+\left(\eta \partial_{x} v\right)^{2}\right) \quad \text { for } y<\frac{3}{2}, \text { i.e., } x<\frac{1}{2},
$$

we can infer

$$
\begin{aligned}
\int_{-1}^{0}\left(1-x^{2}\right)^{\beta} v^{2} \mathrm{~d} x & \lesssim \beta \int_{-1}^{1}\left(1-x^{2}\right)^{\beta+2}\left(\left(v \partial_{x} \eta\right)^{2}+\left(\eta \partial_{x} v\right)^{2}\right) \mathrm{d} x \\
& \lesssim_{\beta} \int_{0}^{\frac{1}{2}} v^{2} \mathrm{~d} x+\int_{-1}^{1}\left(1-x^{2}\right)^{\beta+2}\left(\partial_{x} v\right)^{2} \mathrm{~d} x \\
& \lesssim_{\gamma} \int_{-1}^{1}\left(\left(1-x^{2}\right)^{\gamma} v^{2}+\left(1-x^{2}\right)^{\beta+2}\left(\partial_{x} v\right)^{2}\right) \mathrm{d} x .
\end{aligned}
$$

By symmetry, (B.2) implies estimate (3.3).

Proof of Lemma 3.5. The proof follows the strategy of [17, Lemma 1.2]. For $v \in C^{\infty}([-1,1])$ we have by multiple applications of estimate (3.3) (cf. Lemma 3.2)

$$
\left\|\partial_{x}^{\ell} v\right\|_{L^{2}((-1,1))} \lesssim_{\ell}|v|_{3+2 \ell} \quad \text { and } \quad\left\|\partial_{x}^{\ell} v\right\|_{H^{1}((-1,1))} \lesssim_{\ell}|v|_{5+2 \ell} .
$$

Hence, in view of Definition 1.1 for ||$_{4+2 \ell, *}$, it suffices to establish the estimate (B.3)

$$
\left\|\partial_{x}^{\ell} u\right\|_{C^{0}([-1,1])} \lesssim \int_{0}^{\infty} \inf _{u=u_{-}+u_{+}}\left(\sigma^{-1}\left\|\partial_{x}^{\ell} u_{-}\right\|_{L^{2}((-1,1))}^{2}+\sigma\left\|\partial_{x}^{\ell} u_{+}\right\|_{H^{1}((-1,1))}^{2}\right)^{\frac{1}{2}} \frac{\mathrm{d} \sigma}{\sigma},
$$

where $u_{-} \in H^{\ell}((-1,1))$ and $u_{+} \in H^{\ell+1}((-1,1))$. Writing $w=\partial_{x}^{\ell} u, w_{-}=\partial_{x}^{\ell} u_{-}$, and $w_{+}=\partial_{x}^{\ell} u_{+},($B.3) reduces to

$$
\|w\|_{C^{0}([-1,1])} \lesssim \int_{0}^{\infty} \inf _{w=w_{-}+w_{+}}\left(\sigma^{-1}\left\|w_{-}\right\|_{L^{2}((-1,1))}^{2}+\sigma\left\|w_{+}\right\|_{H^{1}((-1,1))}^{2}\right)^{\frac{1}{2}} \frac{\mathrm{d} \sigma}{\sigma},
$$

where $w_{-} \in L^{2}((-1,1))$ and $w_{+} \in H^{1}((-1,1))$. In fact, since the right-hand side of (B.4) is equivalent to the Besov norm

$$
\|w\|_{B_{1}^{\frac{1}{2}, 2}((-1,1))}=\|w\|_{\left(L^{2}((-1,1)), H^{1}((-1,1))\right)_{\frac{1}{2}, 1}},
$$


(B.3) is a consequence of the embedding $B_{1}^{\frac{1}{2}, 2}((-1,1)) \hookrightarrow C^{0}([-1,1])$, which can be found in [1, Theorem 7.34(b)].

Proof of Lemma 3.6. Again, the proof mainly follows the argumentation of [17, Lemma 1.2]. The second inequality of (3.7) is merely the standard interpolation estimate for the interpolation functor $(\cdot, \cdot)_{*} \sim(\cdot, \cdot)_{\frac{1}{2}, 1}$ that holds independent of the special choice of the underlying norms. We refer to [2, Chapter 5, Proposition 2.10] for a proof.

For the first inequality of (3.7) we use that

$$
\begin{aligned}
|u|_{k, *} \sim\|u\|_{\left(\mathcal{H}^{k-1}, \mathcal{H}^{k+1}\right)_{\frac{1}{2}, 1}} & \gtrsim\|u\|_{\left(\mathcal{H}^{k-1}, \mathcal{H}^{k+1}\right)_{\frac{1}{2}, 2}} \\
& \sim\left(\int_{0}^{\infty} \inf _{u=u_{-}+u_{+}}\left(\sigma^{-1}\left|u_{-}\right|_{k-1}^{2}+\sigma\left|u_{+}\right|_{k+1}^{2}\right) \frac{\mathrm{d} \sigma}{\sigma}\right)^{\frac{1}{2}} .
\end{aligned}
$$

A proof can be found in [2, Chapter 5, Proposition 1.10]. Now we may prove that ${ }^{3}$ $\mathcal{H}^{k}=\left(\mathcal{H}^{k-1}, \mathcal{H}^{k+1}\right)_{\frac{1}{2}, 2}$, i.e., we need to show that

$$
|u|_{k}^{2} \sim_{k}\left(\int_{0}^{\infty} \inf _{u=u_{-}+u_{+}}\left(\sigma^{-1}\left|u_{-}\right|_{k-1}^{2}+\sigma\left|u_{+}\right|_{k+1}^{2}\right) \frac{\mathrm{d} \sigma}{\sigma}\right)^{\frac{1}{2}} .
$$

Therefore, we notice that by induction

$$
\partial_{x}^{k} \mathcal{A}=\mathcal{A}_{k} \partial_{x}^{k} \quad \text { with } \quad \mathcal{A}_{k}=-\left(1-x^{2}\right)^{-k-3} \partial_{x}\left(1-x^{2}\right)^{k+4} \partial_{x}+\left(k^{2}+7 k+10\right),
$$

where $\mathcal{A}$ was defined in (1.22) (compare to Lemma 4.2). This implies that $\mathcal{A}$ is symmetric with respect to all $\langle\cdot, \cdot\rangle_{k}$. As in section 4 (Lemma 4.2, Corollary 4.3) we may then argue that

$$
|u|_{k}^{2} \sim_{k}\left(u, \mathcal{A}^{k} u\right)_{0}
$$

and therefore, instead of (B.5), it suffices to establish

$$
\left(u, \mathcal{A}^{k} u\right)_{0} \sim_{k}\left(\int_{0}^{\infty} \inf _{u=u_{-}+u_{+}}\left(\sigma^{-1}\left(u_{-}, \mathcal{A}^{k-1} u_{-}\right)_{0}+\sigma\left(u_{+}, \mathcal{A}^{k+1} u_{+}\right)_{0}\right) \frac{\mathrm{d} \sigma}{\sigma}\right)^{\frac{1}{2}}
$$

where we may assume $u \in C^{\infty}([-1,1])$ by density (cf. (1.27)). The minimization on the right-hand side of (B.8) can be carried out right away (it is merely a minimization of a quadratic functional in $u_{-}$or $u_{+}$, respectively) and we obtain

$$
u_{+}=\left(1+\sigma^{2} \mathcal{A}^{2}\right)^{-1} u \quad \text { and } \quad u_{-}=\sigma^{2} \mathcal{A}^{2}\left(1+\sigma^{2} \mathcal{A}^{2}\right)^{-1} u .
$$

Note that $\mathcal{A}$ and $\left(1+\sigma^{2} \mathcal{A}^{2}\right)$ are invertible by the Lax-Milgram theorem using (B.7). Applying (B.9) we conclude that the right-hand side of (B.8) can be rewritten as

$$
\begin{aligned}
\int_{0}^{\infty} & \inf _{u=u_{-}+u_{+}}\left(\sigma^{-1}\left(u_{-}, \mathcal{A}^{k-1} u_{-}\right)_{0}+\sigma\left(u_{+}, \mathcal{A}^{k+1} u_{+}\right)_{0}\right) \frac{\mathrm{d} \sigma}{\sigma} \\
= & \left(\left(\int_{0}^{\infty}\left(1+\sigma^{2} \mathcal{A}^{2}\right)^{-1} \mathcal{A} \mathrm{d} \sigma\right) u, \mathcal{A}^{k} u\right)_{0},
\end{aligned}
$$

\footnotetext{
${ }^{3}$ This corresponds to $B_{2}^{k, 2}((-1,1))=W^{k, 2}((-1,1))$ in the unweighted case.
} 
where the bracket in the first argument of $(\cdot, \cdot)_{0}$ is a Bochner integral. We note that $\mathcal{A}$ with domain $D(\mathcal{A}):=\mathcal{H}^{2}$ is a self-adjoint operator in $\mathcal{H}^{0}$ (which mainly follows from (B.6)). By the functional calculus for such operators we may conclude

$$
\int_{0}^{\infty}\left(1+\sigma^{2} \mathcal{A}^{2}\right)^{-1} \mathcal{A} \mathrm{d} \sigma=\int_{0}^{\infty} \arctan (\sigma \mathcal{A}) \mathrm{d} \sigma=\left.\arctan (\sigma \mathcal{A})\right|_{\sigma=\infty}-\left.\arctan (\sigma \mathcal{A})\right|_{\sigma=0} .
$$

It is clear that $\left.\arctan (\sigma \mathcal{A})\right|_{\sigma=0}=0$. We use that $\lambda-\mathcal{A}$ is invertible for every $\lambda \leq 1$ (with the same argumentation as in the context of (B.9)). This implies that by the spectral theorem

$$
\arctan (\sigma \mathcal{A})=\int_{\mathbb{R}} \arctan (\sigma \lambda) \mathrm{d} \mathcal{P}(\lambda)=\int_{1}^{\infty} \arctan (\sigma \lambda) \mathrm{d} \mathcal{P}(\lambda),
$$

where $\mathcal{P}$ is the projection-valued measure associated to $\mathcal{A}$. Since the integration is restricted to $\lambda>1$, by dominated convergence we conclude that

$$
\int_{0}^{\infty}\left(1+\sigma^{2} \mathcal{A}^{2}\right)^{-1} \mathcal{A} \mathrm{d} \sigma=\frac{\pi}{2}
$$

(B.11) in (B.10) shows

$$
\int_{0}^{\infty} \inf _{u=u_{-}+u_{+}}\left(\sigma^{-1}\left(u_{-}, \mathcal{A}^{k-1} u_{-}\right)_{0}+\sigma\left(u_{+}, \mathcal{A}^{k+1} u_{+}\right)_{0}\right) \frac{\mathrm{d} \sigma}{\sigma}=\frac{\pi}{2}\left(u, \mathcal{A}^{k} u\right)_{0},
$$

demonstrating that (B.5) holds true.

Acknowledgments. The author is grateful to Slim Tayachi for inspiring discussions during a visit of the University of Tunis. He also gave valuable advice, in particular on the thin-film literature. Ongoing discussions with Felix Otto and several suggestions of the anonymous reviewers, which have helped to improve the presentation, are appreciated.

\section{REFERENCES}

[1] R. A. Adams and J. J. F. Fournier, Sobolev Spaces, Pure Appl. Math., Elsevier Science, New York, 2003.

[2] C. Bennett and R. Sharpley, Interpolation of Operators, Pure Appl. Math. 129, Academic Press, Boston, 1988.

[3] F. Bernis, Finite speed of propagation and continuity of the interface for thin viscous flows, Adv. Differential Equations, 1 (1996), pp. 337-368.

[4] F. Bernis, Finite speed of propagation for thin viscous flows when $2 \leq n<3$, C. R. Acad. Sci. Paris Sér. I Math., 322 (1996), pp. 1169-1174.

[5] F. Bernis, L. A. Peletier, and S. M. Williams, Source type solutions of a fourth order nonlinear degenerate parabolic equation, Nonlinear Anal., 18 (1992), pp. 217-234.

[6] A. J. Bernoff And T. P. Witelski, Linear stability of source-type similarity solutions of the thin film equation, Appl. Math. Lett., 15 (2002), pp. 599-606.

[7] M. Bertsch, L. Giacomelli, and G. Karali, Thin-film equations with "partial wetting" energy: Existence of weak solutions, Phys. D, 209 (2005), pp. 17-27.

[8] D. Bonn, J. Eggers, J. Indekeu, J. Meunier, And E. Rolley, Wetting and spreading, Rev. Mod. Phys., 81 (2009), pp. 739-805.

[9] E. A. CARLen, Sharp functional inequalities and nonlinear evolution equations, Lecture Notes of the C.I.M.E. Summer School Nonlinear Partial Differential Equations and Applications 2008, in Lecture Notes in Math. 2028, Springer, New York, 2011, pp. 17-86.

[10] E. A. Carlen and S. Ulusoy, Asymptotic equipartition and long time behavior of solutions of a thin-film equation, J. Differential Equations, 241 (2007), pp. 279-292. 
[11] J. A. Carrillo And G. Toscani, Asymptotic $L^{1}$-decay of solutions of the porous medium equation to self-similarity, Indiana Univ. Math. J., 49 (2000), pp. 113-142.

[12] J. A. Carrillo And G. Toscani, Long-time asymptotics for strong solutions of the thin film equation, Comm. Math. Phys., 225 (2002), pp. 551-571.

[13] P. G. De Gennes, Wetting: Statics and dynamics, Rev. Modern Phys., 57 (1985), pp. 827-863.

[14] L. Giacomelli, M. V. Gnann, H. Knüpfer, and F. Otto, Well-posedness for the Navier-slip thin-film equation in the case of complete wetting, J. Differential Equations, 257 (2014), pp. $15-81$.

[15] L. Giacomelli, M. V. Gnann, And F. Otto, Regularity of source-type solutions to the thinfilm equation with zero contact angle and mobility exponent between $3 / 2$ and 3, European J. Appl. Math., 24 (2013), pp. 735-760.

[16] L. Giacomelli and H. KNüPfER, A free boundary problem of fourth order: Classical solutions in weighted Hölder spaces, Comm. Partial Differential Equations, 35 (2010), pp. 2059-2091.

[17] L. Giacomelli, H. KnüPfer, And F. Otto, Smooth zero-contact-angle solutions to a thin-film equation around the steady state, J. Differential Equations, 245 (2008), pp. 1454-1506.

[18] L. Giacomelli and F. Отто, Rigorous lubrication approximation, Interfaces Free Bound., 5 (2003), pp. 483-529.

[19] G. H. HaRdY, Note on a theorem of Hilbert, Math. Z., 6 (1920), pp. 314-317.

[20] D. John, On Uniqueness of Weak Solutions for the Thin-Film Equation, arXiv:1310.6222v1, 2013.

[21] H. KnÜPfer And N. MASmoudi, Darcy flow on a plate with prescribed contact angle: Wellposedness and lubrication approximation, ARMA, to appear.

[22] H. KNÜPFER AND N. MASMOUdi, Well-posedness and uniform bounds for a nonlocal third order evolution operator on an infinite wedge, Comm. Math. Phys., 320 (2013), pp. 395-424.

[23] H. KocH, Non-Euclidean Singular Intergrals and the Porous Medium Equation, Habilitation thesis, University of Heidelberg, 1999.

[24] A. Lunard, Analytic Semigroups and Optimal Regularity in Parabolic Problems, Progr. Nonlinear Differential Equations Appl., Springer, New York, 1995.

[25] D. Matthes, R. J. McCann, And G. Savaré, A family of nonlinear fourth order equations of gradient flow type, Comm. Partial Differential Equations, 34 (2009), pp. 1352-1397.

[26] R. J. McCann and C. SeIs, The Spectrum of a Family of Fourth-Order Nonlinear Diffusions Near the Global Attractor, preprint, 2014

[27] A. MelLet, The thin film equation with non-zero contact angle: A singular perturbation approach, Comm. Partial Differential Equations, 40 (2015), pp. 1-39.

[28] A. Mielke, Über maximale $L^{p}$-Regularität für Differentialgleichungen in Banach- und HilbertRäumen, Math. Ann., 277 (1987), pp. 121-134.

[29] A. Oron, S. H. Davis, And S. G. BAnKoff, Long-scale evolution of thin liquid films, Rev. Modern Phys., 69 (1997), pp. 931-980.

[30] F. Отто, Lubrication approximation with prescribed nonzero contact angle, Comm. Partial Differential Equations, 23 (1998), pp. 2077-2164.

[31] A. PAzy, Semigroups of Linear Operators and Applications to Partial Differential Equations, Appl. Math. Sci. 44, Springer, New York, 1992.

[32] W. Ren And W. E, Boundary conditions for the moving contact line problem, Physics Fluids, 19 (2007), 022101.

[33] C. SEIS, Long-time asymptotics for the porous medium equation: The spectrum of the linearized operator, J. Differential Equations, 256 (2014), pp. 1191-1223.

[34] N. F. Smyth And J. M. Hill, High-order nonlinear diffusion, IMA J. Appl. Math., 40 (1988), pp. $73-86$.

[35] P. K. Suetin, Ultraspherical Polynomials, in Encyclopedia of Mathematics, Springer, New York, 2001. 